Florida International University FIU Digital Commons

2-21-2011

\title{
Performance Enhancement In Accuracy and Imaging Time of a Hand-Held Probe-Based Optical Imager
}

Sergio L. Martinez

Florida International University, smart026@fiu.edu

DOI: $10.25148 /$ etd.FI11051001

Follow this and additional works at: https://digitalcommons.fiu.edu/etd

\section{Recommended Citation}

Martinez, Sergio L., "Performance Enhancement In Accuracy and Imaging Time of a Hand-Held Probe-Based Optical Imager" (2011). FIU Electronic Theses and Dissertations. 397.

https://digitalcommons.fiu.edu/etd/397 


\title{
FLORIDA INTERNATIONAL UNIVERSITY
}

Miami, Florida

\section{PERFORMANCE ENHANCEMENT IN ACCURACY AND IMAGING TIME OF A HAND-HELD PROBE-BASED OPTICAL IMAGER}

\author{
A thesis submitted in partial fulfillment of the \\ requirements for the degree of \\ MASTER OF SCIENCE \\ in
}

BIOMEDICAL ENGINEERING

by

Sergio L. Martinez 
To: Dean Amir Mirmiran

College of Engineering and Computing

This thesis, written by Sergio L. Martinez, and entitled Performance Enhancement in Accuracy and Imaging Time of a Hand-Held Probe-Based Optical Imager, having been approved in respect to style and intellectual content, is referred to you for judgment.

We have read this thesis and recommend that it be approved.

$\begin{array}{r}\hline \text { Armando Barreto } \\ \hline \text { Wei-Chiang Lin } \\ \hline \text { Anuradha Godavarty, Major Professor }\end{array}$

Date of Defense: February 21, 2011

The thesis of Sergio L. Martinez is approved.

Dean Amir Mirmiran

College of Engineering and Computing

Interim Dean Kevin O'Shea

University Graduate School

Florida International University, 2011 


\section{ABSTRACT OF THE THESIS \\ PERFORMANCE ENHANCEMENT IN ACCURACY AND IMAGING TIME OF A HAND-HELD PROBE-BASED OPTICAL IMAGER}

by

Sergio L. Martinez

Florida International University, 2011

Miami, Florida

Professor Anuradha Godavarty, Major Professor

The Optical Imaging Laboratory has developed a hand-held optical imaging system that is capable of $3 \mathrm{D}$ tomographic imaging. However, the imaging system is limited by longer imaging times, and inaccuracy in the positional tracking of the handheld probe. Hence, the objective is to improve the performance of the imaging system by improving imaging time and positional accuracy. This involves: (i) development of automated single Labview-based software towards near real-time imaging; and (ii) implementation of an alternative positional tracking device (optical) towards improved positional accuracy during imaging. Experimental studies were performed using cubical tissue phantoms (1\% Liposyn solution) and 0.45-cc fluorescence target(s) placed under various conditions. The studies demonstrated a $90 \%$ reduction in the imaging time (now $\sim 27 \mathrm{sec} /$ image) and also an increase from $94 \%$ to $97 \%$ in the positional accuracy of the hand-held probe. Performance enhancements in the hand-held optical imaging system have improved its potential towards clinical breast imaging. 


\section{TABLE OF CONTENTS}

CHAPTER

PAGE

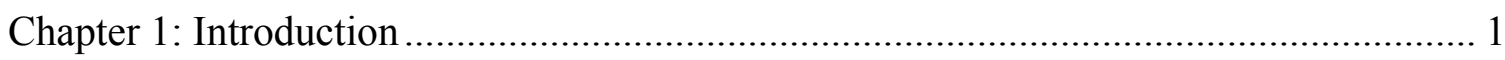

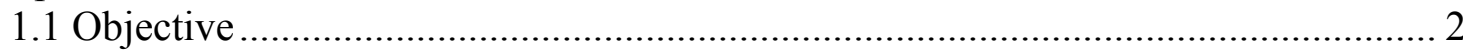

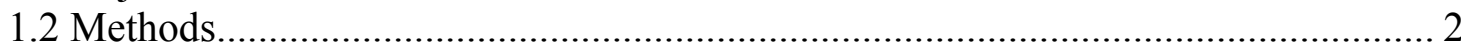

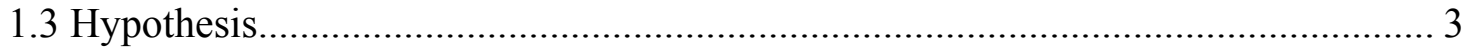

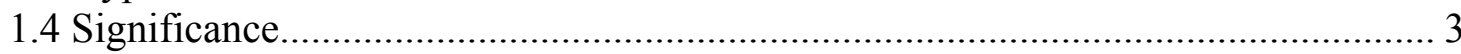

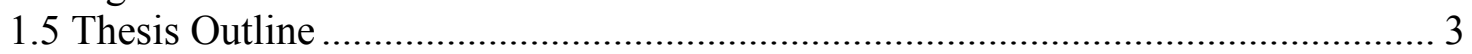

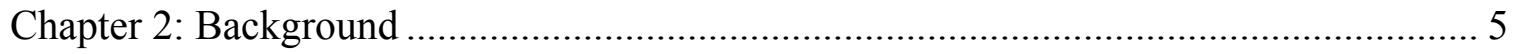

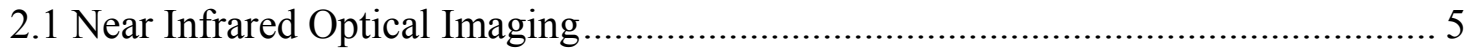

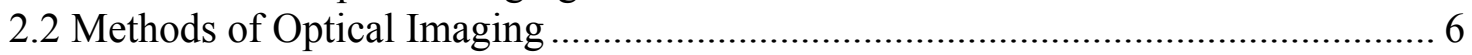

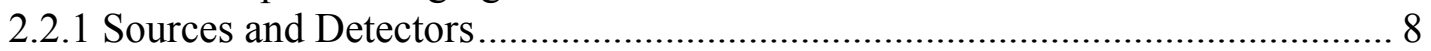

2.2.2 Techniques of Optical Imaging................................................................. 9

2.3 Clinical Translation of Optical Imaging ............................................................ 11

2.3.1 Hand Held Optical Imagers ......................................................................... 12

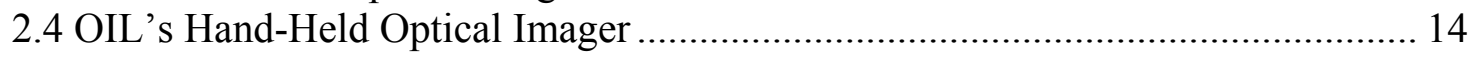

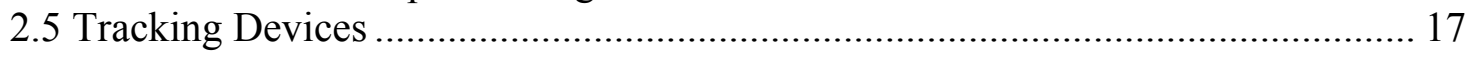

2.6 Co-registration with OIL's Optical Imager.......................................................... 21

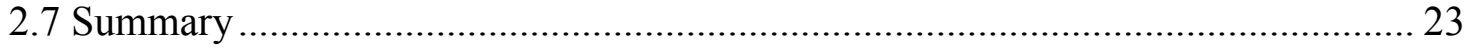

Chapter 3: Materials and Methods ............................................................................... 26

Part I Modifications Towards the First Generation Optical Imager ............................. 26

3.1 Automation of Co-registration........................................................................ 26

3.2 Study I: Experimental Evaluation of the Automated Software ............................. 29

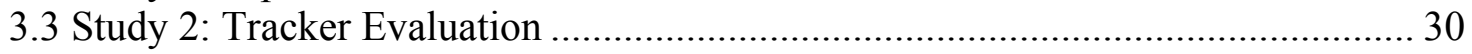

3.3.1 Study I: Effectiveness of three trackers towards 2D positioning ..................... 30

3.3.2 Electromagnetic Tracker Analysis ............................................................... 30

3.3.3 Acoustic Tracker Analysis...................................................................... 32

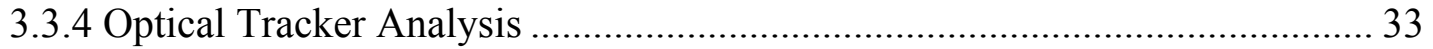

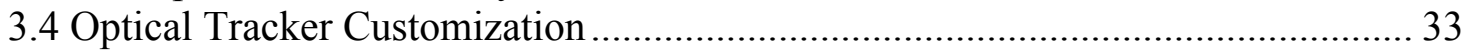

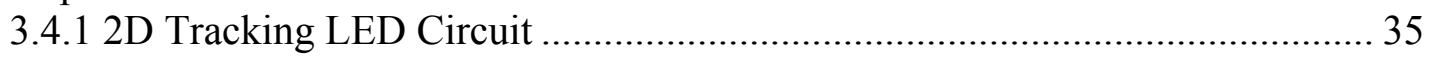

3.4.2 Converting Wiimote Raw Data to Distances ................................................... 36

3.4.3 Operational Limitations of the Wiimote .......................................................... 39

3.4.4 Optical Tracker 2D Integration to Automated Software.................................. 40

3.4.5 2D Co-registration Testing with Tracker ......................................................... 43

3.4.6 Optical and Acoustic Tracker 2D Co-registration with Probe.......................... 44

3.4.7 Fine Movement Tracking with Optical Tracker .............................................. 46

3.5 Customization of Optical Tracker for 3D Tracking.............................................. 46

3.6 Optical Tracker LED Affect on Imaging ................................................................ 46

Part II Modifications Towards Second Generation Optical Imager .............................. 47

3.7 Second Generation Optical Imaging Device........................................................... 47 


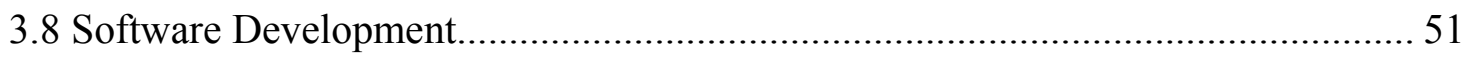

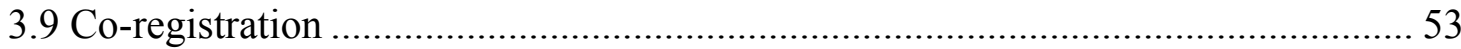

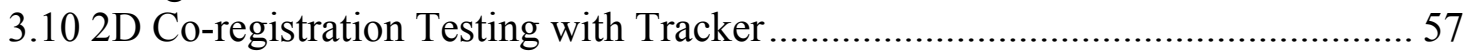

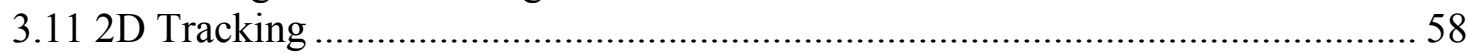

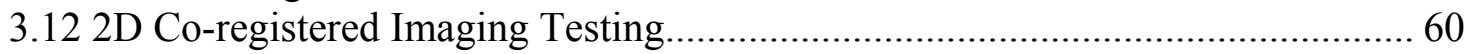

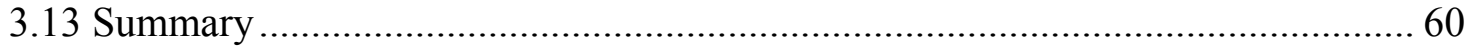

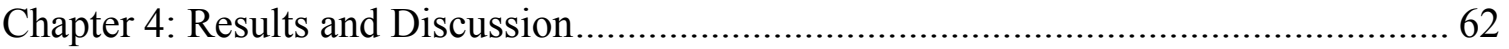

Part I Modifications Towards the First Generation Optical Imager .............................. 62

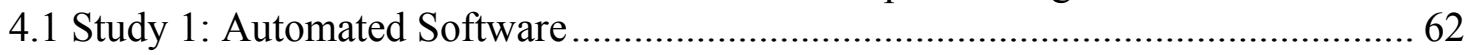

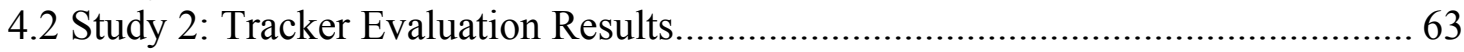

4.2.1 Electromagnetic vs. Acoustic Tracking Results ............................................... 63

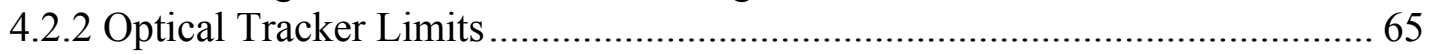

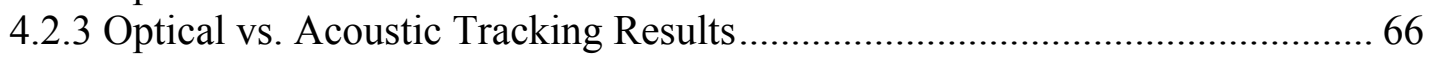

4.3 Optical and Acoustic Tracker 2D Co-registration .................................................. 68

4.4 Optical Tracking LED Affect on Imaging .......................................................... 72

Part II Modifications Towards Second Generation Optical Imager ............................. 73

4.5 Second generation Optical Imager............................................................................ 73

4.6 Co-registration with the Second Generation Optical Imager................................... 77

4.7 2D Tracking with the Second Generation Optical Imager....................................... 78

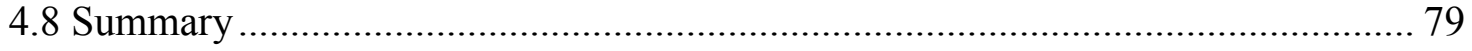

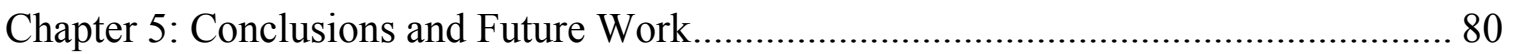

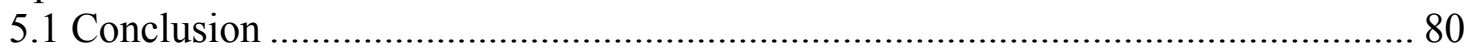

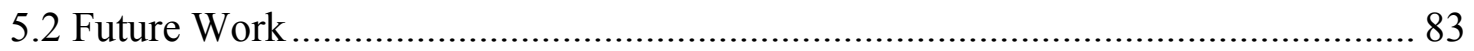

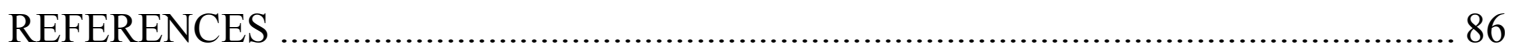

APPENDIX 


\section{LIST OF TABLES}

TABLE

PAGE

Table 2.1 Comparison of trackers towards tomographic imaging with OIL's hand held probe shows that optical tracking may provide to be the most

effective.

Table 3.1 The comparison of features between first and second-generation optical imagers.

Table 3.2 Comparison of features of the first and second-generation co-registration software

Table 3.3 Positions collected in the 2D tracking study for the second generation probe heads. Both probe heads were moved in the same positions on separate phantoms where $(0,0)$ is the bottom right position of the phantom. Therefore each of these positions was repeated 5 times per probe head.

Table 4.1 Error comparison of the tracking devices in two dimensional tracking. The averages of the errors are shown here as well as the average distance from the true measured position as calculated using Equations 3 -

6.

Table 4.2 Comparison of the target location and errors of the probe location for both trackers. The location of the target was taken from the images that were collected in the $2 \mathrm{D}$ studies with a $2 \%$ Liposyn filled cubical phantom. The true location of the target was compared to the location found in the images giving the errors shown above.

Table 4.3 Table showing the error of the acoustic, optical, and multiple objects with optical. Each of the co-registration software's accuracy was compared in a study where the probe head with the tracker attached was moved along the face of a cubical phantom. The positions at which the probe(s) head was/were located were measured physically and through the co-registration software to provide an accurate comparison of the accuracy of each

tracker. 


\section{LIST OF FIGURES}

FIGURE

PAGE

Figure 2.1 Time domain signal showing an input signal for tissue imaging. Image collection is defined as a Temporal Point Spread Function as shown above.

Figure 2.2 Frequency domain signal showing a sinusoidal input signal for tissue imaging.

The signal collected also has a sinusoidal shape, however phase shifted. 10

Figure 2.3 Continuous wave signal shown is a simple non-modulated source signal for tissue imaging. The detected output light is typically of lower intensity. 11

Figure 2.4 Current optical imaging devices. A above is a large bed manufactured by Imaging Diagnostic Systems, Inc. It involves the breast to be imaged through transillumination while placed in a hole on the imaging bed. B above is also a transillumination imager that requires compression of the breast for imaging [Wang, et al, 2010]. C above is a hand held imager, however is for spectroscopic measurements [Bevilacqua, et al, 2000]]

Figure 2.5 Hand held optical imagers currently in the research stage. A is a round hand held imager containing 8 sources and a single detector [Chance et al, 2005]. B and C above are both hand held imagers involving optical and acoustic imaging in combination with one another [Zhu et al, 1999] ............................................... 13

Figure 2.6 First Generation Hand-Held Probe Head [Jayachandran, et al. 2007] 15

Figure 2.7 OIL's hand held optical imager and a blank phantom where (i) is the source, (ii) is the detector and (iii) is the hand held probe head in contact with a blank phantom [Jayachandran, et al. 2007] 16

Figure 2.8 Acoustic Tracking Device (Logitech tracker manual, Logitech Inc., Fremont, CA.).

Figure 2.9 Graphical depiction of the co-registration software's function in (A) obtaining optical data from WinView and (B) positional data from MatLab simultaneously. The data is then (C) super imposed in LabView to obtain the (D) co-registered image on a mesh of the surface.

Figure 3.1 Graphical user interface of the new automated software. The focus window on the right shows the raw image from the camera. Once the "Acquire Image" button is pressed the 2D image collected is displayed. 28

Figure 3.2 Flowchart of the software. (A) is where the previous software was run through WinView and has now been integrated with (B) the LabView based co-registration software for full automation. 
Figure 3.3 Polhemus Fastrak Electromagnetic Tracking Device. (A) is the transmitter, (B) is the receiver, and $(\mathrm{C})$ is the control box

Figure 3.4 Representation of the cubical phantom with the labeled points of where the tracker receiver was placed

Figure 3.5 Logitech Ultrasound 3D Head Tracker

Figure 3.6 Nintendo Wii controllers used as a tracking device where (a) are the Wii controllers, (b) are the tracked infrared LED's, and (c) is the Bluetooth waves from the Wii controllers that allow wireless communication with (d) the host computer. 34

Figure 3.7 Flowchart of Wiimote Data Collection Process ......................................... 35

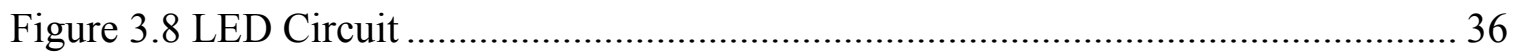

Figure 3.9 Setup for the 2D tracking equation. The Wiimote was placed at multiple distances from the $(\mathrm{x}, \mathrm{y})$ plane where the LED was placed. The LED was then moved in the $\mathrm{x}$ and $\mathrm{y}$ direction to determine the equation to convert the pixel values to distances.

Figure 3.10 Sample of one data set of collected positions to determine the formula to convert pixels to $\mathrm{cm}$. As can be seen the $\mathrm{R}^{2}$ value average for all samples collected was .99982 with standard deviation of $8.32 \mathrm{E}-05$. The plots show a linear relation between the measured LED position and the pixel value given by the camera. The black line represents the line of best fit.

Figure 3.11 The angle for field of view was measured using simple geometry. 40

Figure 3.12 Flowchart for Wiimote Tracking Integrated into the Co-registration Software

Figure 3.13 Setup for Tracker and Co-Registration Testing.

Figure 3.14 The figure depicts the motion that was performed on the large $20 \times 20 \times 20 \mathrm{~cm}$ phantom with the probe head and tracker attached to obtain the location of the target in the images collected. A $2 \%$ Liposyn phantom and a $1 \mu \mathrm{M}$ indocyanine green (ICG) $.45 \mathrm{cc}$ target were used. The arrows show the direction of the of the movements of the probe head

Figure 3.15 Depiction of how the effect of the LED on imaging was performed where a $2 \%$ Liposyn phantom was used and the LED was placed on the surface of the phantom and on the center of the probe head to see if the IR signal from the LED effects the images collected. 
Figure 3.16 Second Gen Probe Head (left) and the first generation probe head (right). Although the second generation probe heads may be smaller individually, they have more flexibility and are one flexible piece on the probe face allowing for maximum contact while the first generation optical imager is three individual solid plates.... 48

Figure 3.17 Second generation System in its Cart (left) compared to the first generation optical imager (right). It can easily be seen that the second-generation system is much more compact and portable than the bench top first generation optical imager.

Figure 3.18 Second Generation Co-Registration Approach Software Front Panel 57

Figure 4.1 Comparison of the results obtained from the electromagnetic tracker and the acoustic tracker. The transmitter end of each tracker was moved along the four corners of the face of a cubical phantom.

Figure 4.2 Comparison of tracked positional information using an optical and acoustic tracker. The LED (optical tracker) and transmitter (ultrasound tracker) were moved in a linear fashion along the face of a cubical phantom. Positions were recorded at .5 $\mathrm{cm}$ increments.

Figure 4.3 Co-registered imaging demonstrating the detection of a target as the probe head is moved in different positions along the face of a cubical phantom. The black circle represents the position of the.45 cc ICG target within a $2 \%$ Liposyn filled cubical phantom. The dotted black box is the outline of the probe head.

Figure 4.4 2D Contour Images of intensity signals acquired from the phantom surface under the following conditions: (A) When the LED located on the hand-held probe were turned off, (b) Comparing the effect of the Tracker's LED On and Off. The LED off image involved the LED being attached to the probe but not powered while the LED on image involved the LED being turned on while attached to the probe. The subtracted image involves the subtraction of the LED on image from the LED off image giving the difference of the two images.

Figure 4.5 2D surface contour plots of intensity distribution acquired on two $10 \mathrm{x} 10 \mathrm{x}$ $10 \mathrm{cc}$ cubical phantoms using the 2 probe heads of the Gen- 2 imager. A $0.45 \mathrm{cc}$ absorption contrasted target filled with $.08 \%$ by volume India Ink was placed in Image collected with the second generation imaging system. The top image displays the images taken with the target in place. The center images display the images taken of the phantom without the target in place. Finally the subtracted images show the lesion location once the background is subtracted from the target. 76 
Figure 4.6 Co-registered images from the second generation optical imager. The image on the left corresponds to probe 1, and the right to probe 2. Each probe was on a separate cubical phantom and both images were collected with the same camera at the same time. The image is a previously recorded image and does not represent any signal coming from the probe heads or the phantom. 


\title{
ACRONYMS
}

\author{
1D One Dimensional \\ 2D Two Dimensional \\ 3D Three Dimensional \\ APD Avalanche Photodiode \\ CCD Charge-Coupled Device \\ CW Continuous Wave \\ CT Computed Tomography \\ DOI Diffuse Optical Imaging \\ EM Electromagnetic \\ FD Frequency Domain \\ FDPM Frequency Domain Photon Migration \\ FIU Florida International University \\ GUI Graphical User Interface \\ ICCD Intensified Charge-Coupled Device \\ ICG Indocyanine Green \\ LED Light Emitting Diode \\ MRI Magnetic Resonance Imaging \\ NIR Near-Infrared \\ OIL Optical Imaging Laboratory \\ PET Positron Emission Tomography \\ PMT Photon Multiplier Tube \\ TD Time Domain
}




\section{Chapter 1: Introduction}

Near-infrared optical imaging is a promising, non-invasive technology with the potential of diagnostic breast imaging. In recent years there has been much research towards the clinical translation of bulky optical imagers, as well as the development of hand held optical imagers. However, current hand-held optical imagers are only capable of two-dimensional (2D) spectroscopic surface images and cannot perform threedimensional (3D) tomography [Chance, et al. 2005; Chen, et al. 2004; No, et al. 2005; Sao, et a. 2003; Tromberg, et al. 2005; Xu, et al. 2007; Zhu, et al. 1999]. Recently, the Optical Imaging Laboratory (OIL) has developed a hand held optical imaging system that is capable of 3D tomography. To obtain measurements relevant for 3D tomographic imaging, a co-registration technique is employed, that allows registering the positional information of the hand-held probe on the tissue geometry during imaging studies. The feasibility of co-registered imaging and 3D tomography studies on tissue phantoms have been demonstrated in the past. [Ge, et al. 2008; Jayachandran, et al. 2007]. However, there are limitations in our hand-held optical imaging system in the path of our ongoing clinical translational efforts. These include:

(i) Longer imaging times: The optical imaging system and the positional tracking device are operated independently via their respective software. This is computationally cumbersome and also tends to slow the overall imaging time.

(ii) Instability of the positional tracking device: The ultrasound-based tracking device used to obtain 3D positional information of the hand-held probe during imaging is unstable, thus causing inaccuracies in the $3 \mathrm{D}$ positional data. This 
in turn tends to impact the location of the detected target during $2 \mathrm{D}$ and $3 \mathrm{D}$ imaging studies.

\subsection{Objective}

The objectives of my thesis involve the reduction of the overall imaging times as well as improve the stability and accuracy of the positional tracking device.

\subsection{Methods}

The methods used to achieve the objective are to:

(i) Develop an automated co-registration technique that can operate both the optical imaging system and the positional tracking device simultaneously via a single LabView based software. This allows decrease in the overall imaging times as well as makes it user-friendly to perform imaging studies.

(ii) Implement an alternative positional tracking device (optical over ultrasound) and also develop the appropriate software relevant to the tracking device towards automated co-registered imaging. This allows improved stability and accuracy of the positional data during imaging studies, apart from being a cost-effective device.

The automated co-registration software as well as the alternate positional tracking device were developed and implemented into two generations of our optical imaging system. 


\subsection{Hypothesis}

The hypothesis is twofold: (i) Simultaneous operation of multiple tasks during imaging (e.g. image acquisition, positional tracking, and image processing) can significantly reduce the overall imaging time, thus allowing a near real-time imaging in a clinical environment. Additionally, by operating the entire imaging system (including the positional tracking device) using single software, makes the imaging process userfriendly in a clinical setting. (ii) Implementation of an alternate tracking device that is more stable, accurate and cost-effective can improve the accuracy of co-registered imaging, which in turn can improve the quality of target detection.

\subsection{Significance}

The clinical application of the optical imaging device will complement currently employed imaging tools used towards breast cancer diagnosis. By automating the software, the image acquisition time is decreased to a near real-time imaging approach, which will allow for clinicians to acquire extensive images with least patient time during the diagnostic imaging procedure. By improving the effectiveness of the tracking device, the accuracy in tumor detections can potentially improve, thus preventing invasive methods of locating tumors that are falsely identified.

\subsection{Thesis Outline}

In chapter 2, the Background of optical imaging and a brief overview of currently available hand-held based optical imagers are described. Additionally, the Optical Imaging Laboratory's custom built hand held optical imaging system (first generation) is introduced, detailing unique features and shortcomings. Immediately following is a 
description of the tracking devices and the concept of co-registration. Chapter 3 is the Materials and Methods of automation of the entire imaging process, and the development and testing of a more accurate and stable tracking device. The development of a second generation hand-held optical imaging system (carried out as a team effort in the laboratory) and the implementation of the developed automated software and the tracking device are also described. Chapter 4 follows with the Results and Discussion of tissue phantom studies that demonstrate near real-time imaging using the automated coregistration software and the alternate positional tracking device. Additionally, the accuracy of the positional tracking device and its effectiveness in 2D target detectability is assessed and compared to the past tracking device. The experimental studies were performed on both the first and second generation hand-held optical imaging systems. Finally, chapter 5 concludes the thesis and summarizes the future direction of the work. 


\section{Chapter 2: Background}

\subsection{Near Infrared Optical Imaging}

Breast cancer is the most highly diagnosed cancer in women in the world with an estimated 207,090 new cases in 2010[American Cancer Society, 2010]. Although treating breast cancer is highly important, preventative techniques, such as early diagnosis, are highly valuable in the research community. Current methods of diagnosis include x-ray, x-ray computed tomography (CT), ultrasound, positron emission tomography (PET), and magnetic resonance imaging (MRI). Although for many years these diagnosis techniques have proven to be effective methods of imaging with high sensitivity they suffer from various drawbacks such as

- Radiation from X-Ray and CT can cause harmful effects over prolonged/multiple uses

- High discomfort used for compressing the breast in X-Ray, CT, and ultrasound

- Ionization caused by PET can be harmful to the patient

- Poor resolution in ultrasound can make it difficult for doctors and researchers to determine if and where lesions are present in the tissue

- High cost of MRI causes many people to avoid MRI if they cannot afford it

- Poor specificity of all these modalities may cause a high number of false positives. 
In order to improve on these diagnostic modalities, there is a desire for a less invasive, non-ionizing, high resolution and high specificity/sensitivity imaging technique that is low cost. One technology that has the potential of achieving these objectives is near infrared imaging (NIR).

Near-infrared imaging or optical imaging is a promising non-invasive, nonionizing technology for breast cancer diagnosis among countless other uses, such as functional brain mapping and oximetry. NIR uses the therapeutic window of light, 700$900 \mathrm{~nm}$ wavelength, where light is highly scattered and minimally absorbed in the tissue allowing for tissue characterization through their optical properties. Being minimally absorbed and highly scattered, NIR can also allow for deep tissue imaging giving functional information of biological systems. Although NIR has inherent absorptive properties in tissue, different tissue have different absorptive properties giving an NIR image contrast, which can be further be improved with fluorescing agents to more accurately improve the quality of a collected image using NIR.

\subsection{Methods of Optical Imaging}

Optical imaging (or NIR imaging), in general, can be performed through many different methods such as spectroscopy and diffuse optical imaging (DOI) among others. Spectroscopic imaging of breast tissue involves the launching of source light into the breast tissue where the reemitted light is then collected by a detector, typically a photodetector, photon multiplier tube (PMT) or avalanche photodiode (APD). Spectroscopy can measure the oxygen content of the hemoglobin in an area of interest thus giving functional information and optical properties of the area through investigation 
of the spectra using an inverse algorithm. Diffuse optical imaging refers to the optical imaging of biological tissue in the diffusive regime [Biomedical Optics 2007. DOI systems can be placed in many different source and detector configurations depending on its application for imaging an object, in this case tissue. Image reconstruction in DOT involves both forward and inverse algorithms. In the forward algorithm, the distribution of reemitted light can be predicted based on presumed parameters for the source and object of interest. In the inverse algorithm, the distributions of the optical properties of the object of interest are reconstructed from a measured data set, respectively.

Breast imaging can be performed through configurations including planar transmission or planar reflection [Biomedical Optics 2007]. In general a source will illuminate the area of interest and the detectors will measure the reflected or transmitted light, depending on the configuration. Transmitted light refers to the light that travels through the tissue and is generally collected on the opposite side from where the light is launched. However, transmitted light can also be collected in other areas that are not directly in the same face at which the light was launched into the tissue. Reflected light is that light which is collected on the same face at which the light was launched. Reflectance does not necessarily mean the light hits the surface and reflects back, but it travels through the tissue and scatters in such a manner that it returns to the same face at which the light was launched. Subsequently a computer will take the information collected from the detector and reconstruct the data using complex mathematical techniques. The reconstructed data collected from the tissue will contain a 2D or 3D image mapping the hemoglobin concentrations in the tissue. 


\subsubsection{Sources and Detectors}

In the case of NIR or optical imaging, the wavelengths fall between $700-900 \mathrm{~nm}$ since light in the tissue is minimally absorbed and preferentially scattered. In order to illuminate the area of interest laser diodes are typically used which emit a particular wavelength of interest. Laser diodes can be placed either in contact with the tissue or illuminate from a particular distance from the tissue producing a more disperse illumination method. Also, in order to transfer the light from one point to another for most systems, optical fibers are used. Although optical fibers can be used to send light from a source laser diode to the tissue, they are also used as a mechanism to transfer light from the tissue surface to the detector. Other methods of illumination include area illuminating white light and LED lighting. With white light the area of interest is illuminated with a halogen or xenon light source, which is usually used in spectral imaging in multiple wavelengths. Optical filters are used on the detector end to collect the wavelengths of interest.

Detectors in optical imaging include photon multiplier tubes (PMT), avalanche photodiodes (APD), and charge coupled devices (CCD). PMTs work in a fashion that multiplies the current produced by incident light in the order of $10^{6}$, in multiple dynode stages, allowing for individual photons to be detected when the incident light is very low. APDs are a highly sensitive semiconductor electronic device that converts light to electricity by applying a high reverse bias voltage. CCDs work in a fashion where the CCD captures a light signal, which charges the capacitors of the CCD. The charge is then transferred through capacitive bins that manipulate the charge in a manner that converts the light into a digital signal. Typically in optical imaging the light signal is very low. In 
order to compensate for the low signal, an image intensifier is used with the CCD thus called an intensified charge coupled device (ICCD).

\subsubsection{Techniques of Optical Imaging}

Optical imaging can be classified into three different techniques depending on the type of signal, which are time domain (TD), frequency domain (FD), continuous wave (CW). Time-domain (TD) mode involves a source emitted as an ultra short pulse of light, in the order of picoseconds, into a scattering object as shown in figure 2.1. The reemitted light signal's response can be resolved through the use of a single photon counting system or streak cameras. The light is collected by a detector such as a CCD, APD, or PMT. Of the three modes, TD contains the most information about the tissue but is also the slowest and most expensive [Wang et al, 2007].

\section{Time Domain}

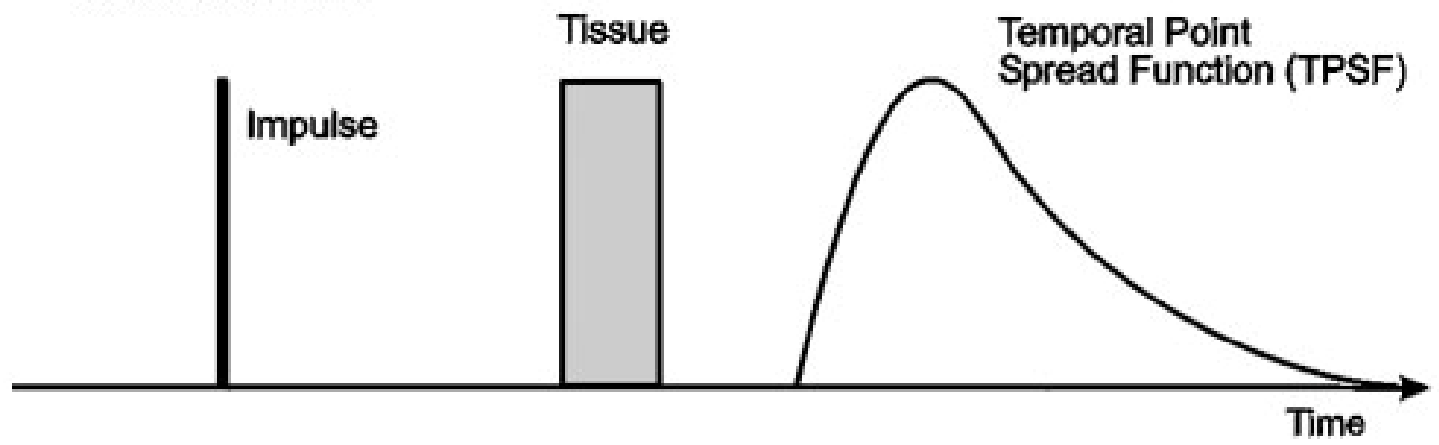

Figure 2.1 Time domain signal showing an input signal for tissue imaging. Image collection is defined as a Temporal Point Spread Function as shown above.

In FD mode, the emitted light source is amplitude modulated and the transmitted/reflected light modulation has a reduced modulation depth as shown in figure 2.2 [Wang et al, 2007]. Typically the output power of a laser diode source is modulated 
at a particular frequency from a signal generator and emitted through the tissue. The transmitted/reflected light is collected by the detector, which is modulated as well. Since both source and detector are modulated in frequency domain, the data can be collected via homodyne or heterodyne technique. The homodyne technique is where the source and detector are modulated at the same frequency while the heterodyne technique involves different frequencies for source and detector. The collection of information through FD mode includes amplitude and phase of the signal, which can inherently describe the scattering and absorption coefficients of the tissue through detectors such as PMT, APD, or CCD. FD mode, although contains less information than TD mode, it is much faster and less expensive.

\section{Frequency Domain}

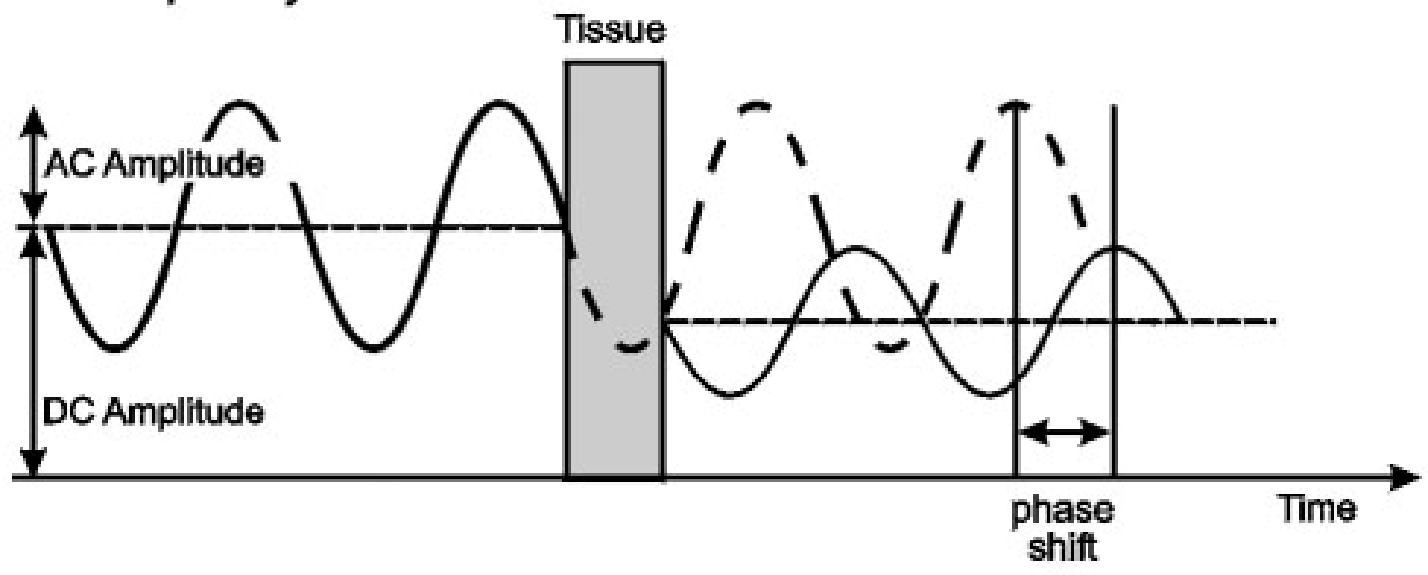

Figure 2.2 Frequency domain signal showing a sinusoidal input signal for tissue imaging. The signal collected also has a sinusoidal shape, however phase shifted.

In $\mathrm{CW}$ mode, the source light is time-invariant and contains no direct information about the time of flight of the signal [Wang et al, 2007]. The light is emitted by a laser diode source into the tissue where the reemitted light as shown in figure 2.3 , and is then 
collected by either a PMT, APD, or CCD. Although the CW mode does not contain no direct information to separate the absorption and scattering, it is the fastest and least expensive of the three modes.

\section{Continuous Wave}

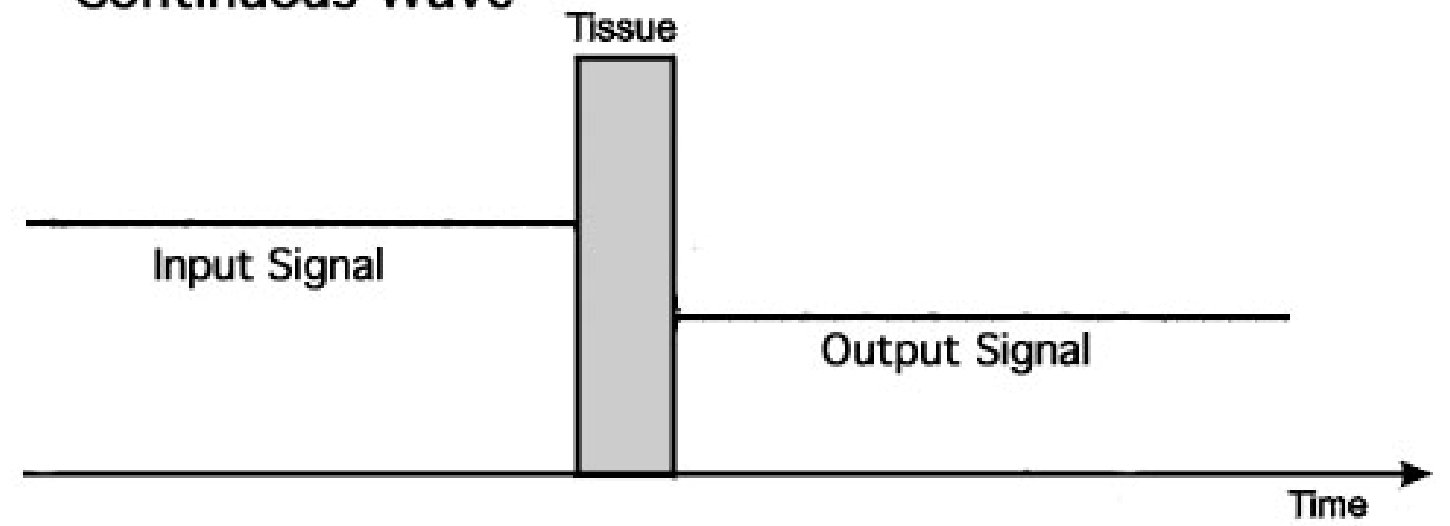

Figure 2.3 Continuous wave signal shown is a simple non-modulated source signal for tissue imaging. The detected output light is typically of lower intensity.

\subsection{Clinical Translation of Optical Imaging}

Currently, research involving DOI has seen an increase in the clinical translation of this technology using either bulky bench top or hand-held based optical imagers (examples shown in figure 2.4) [Erickson, et al. 2008, Chance, et al. 2005; Chen, et al. 2004; No, et al. 2005; Sao, et a. 2003; Tromberg, et al. 2005; Xu, et al. 2007; Zhu, et al. 1999] for various applications. The larger bench top imagers typically include either a mechanism in which the subject lies face down where the breast is placed in a (semispherical) hole containing the source and detector for transmittance configuration of imaging or involving configurations for either transmittance or reflectance configurations with compression applied to the breast. Hand-held imagers are smaller and may or may 
not require the application of pressure to the breast, however they have mostly involved in spectroscopic imaging and few tomographic imagers.

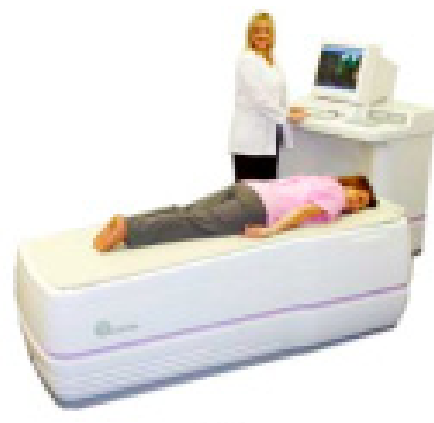

A

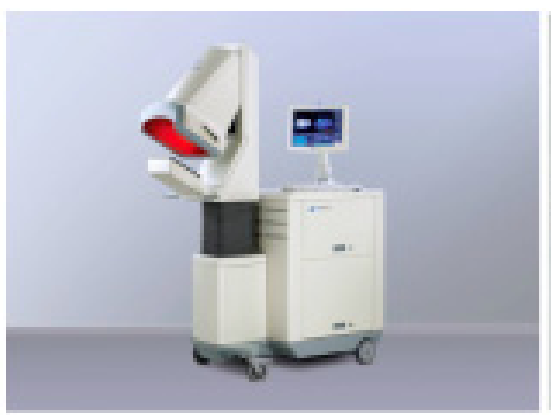

B

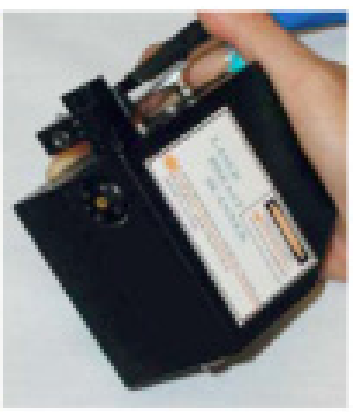

C

Figure 2.4 Current optical imaging devices. A above is a large bed manufactured by Imaging Diagnostic Systems, Inc. It involves the breast to be imaged through transillumination while placed in a hole on the imaging bed. B above is also a transillumination imager that requires compression of the breast for imaging [Wang, et al, 2010]. C above is a hand held imager, however is for spectroscopic measurements [Bevilacqua, et al, 2000]].

\subsubsection{Hand Held Optical Imagers}

Current hand-held optical imagers (shown in figure 2.5) are primarily able to perform surface scans and provide spectroscopic information, but cannot perform threedimensional (3D) tomography ${ }^{1}$ [Chance, et al. 2005; Chen, et al. 2004; No, et al. 2005; Sao, et a. 2003; Tromberg, et al. 2005; Xu, et al. 2007; Zhu, et al. 1999].

\footnotetext{
${ }^{1}$ A method of imaging involving taking multiple images as slices of the whole body and then reconstructing the image from the slices.
} 


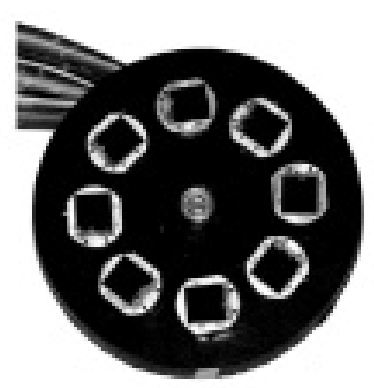

A

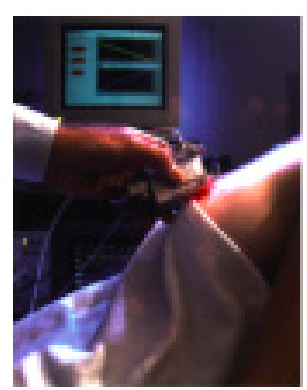

B

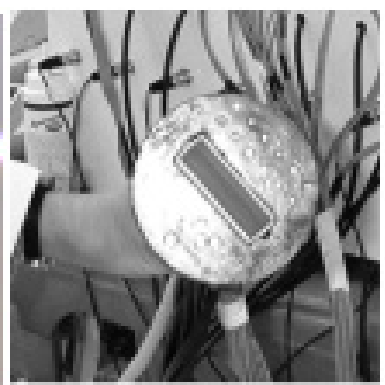

C

Figure 2.5 Hand held optical imagers currently in the research stage. A is a round hand held imager containing 8 sources and a single detector [Chance et al, 2005]. B and C above are both hand held imagers involving optical and acoustic imaging in combination with one another [Zhu et al, 1999].

The type of data that a hand held imager can collect is determined by the configuration of the source and detectors. A single source and detector pair will collect spectroscopic data for simple characterization at a particular point. Tomography usually requires multiple sources and detectors to characterize volumetric information by imaging in sections and then a reconstruction algorithm is used to display a 3D image of the collected slices or images. For these imaging techniques the source and detectors make contact with the breast tissue via a probe head, which is typically the hand held portion of the imager.

Other research groups have based their imaging approach on the photoacoustic methods [Zhu, et al. 1999], which combine ultrasound with the optical data. They obtain the physical information about the tissue as well as the depth aspect of the signal through an ultrasound imaging system while the optical sources and detectors that surround the ultrasound system also collect optical data from the tissue. The optical data is then coregistered with the ultrasound to combine the functional (from the optical data) with the 
structural information (from the ultrasound data) and locate a tumor in the tissue [Zhu, et al 2010]. Other groups have developed FD and CW NIR spectroscopic imagers that are capable of collecting both the FD and CW simultaneously allowing for more information about the tissue than just FD or CW alone [Cerussi, et al, 2006; Cerussi, et al, 2007; Erickson, et al 2008], allowing for less post processing to acquire the tissue information. While yet other research groups have also included fluorophores in the tissue or phantoms to enhance the contrast of the image [Erickson, et al 2008].

Although various researchers have developed hand-held probe based imagers with multiple sources and detectors, the probe heads were smaller and the studies were more focused on 2D spectroscopic imaging. Other researchers have yet to successfully combine optical imaging with 3D tomography capabilities in a hand held based system. However in our Optical Imaging Laboratory (OIL), a hand held probe was developed, capable of 3D tomography with multiple sources and detectors placed on a large probe head, towards diagnostic breast imaging studies. OIL is the first to combine simultaneous illumination and detection in a multi-source, multi-detector tomographic hand held imager.

\subsection{OIL's Hand-Held Optical Imager}

The hand held optical imager developed in our laboratory (OIL) is based on the homodyne imaging approach. It consists of three major components; (i) the laser diode source (ii) an ICCD detector and (iii) the hand held probe [Ge, et al. 2008; Jayachandran, et al. 2007] as can be seen in figure 2.6. The hand held probe seen in figure 1.6 consists of 165 detector fibers, 6 source fibers and a 3-piece face capable of contouring to curved 
surfaces with tissue surface contact. The source fibers are connected to a collimated $785 \mathrm{~nm}$ laser diode source, while the detector fibers are coupled to the ICCD and focused through a focusing lens. The optical imager is capable of collecting both FD and CW measurements. The source is temperature and current controlled and is coupled to a frequency synthesizer and amplifier for modulation. In order to perform FD optical imaging, the ICCD is also coupled with a frequency synthesizer and amplifier for modulation.

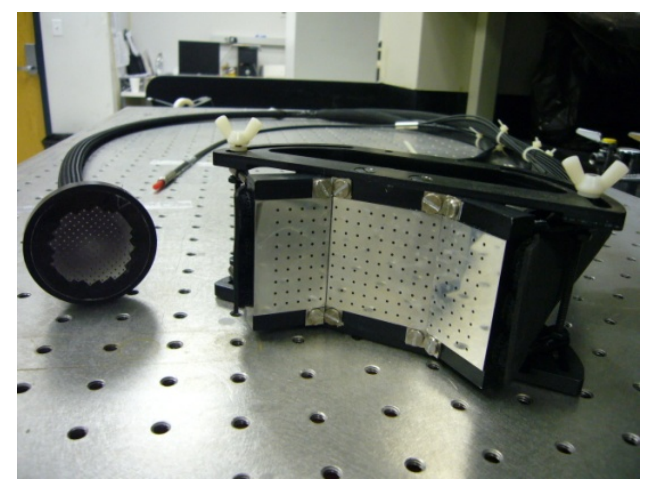

Figure 2.6 First Generation Hand-Held Probe Head [Jayachandran, et al. 2007]

The hand held optical imager has the following unique features:

1. Probe head that can contour to tissue curvatures: Uses a three piece probe face that flexes as seen in figure 2.6 [Jayachandran, et al. 2007].

2. Rapid imaging over large areas: Uses a multi-source illumination technique with simultaneous detection [Jayachandran, et al. 2007].

3. 3D tomography: Simultaneously tracks 3D positional information and optical data co-registered onto a mesh of the imaged surface [Ge, et al. 2008; Ge, et al. 2009]. 


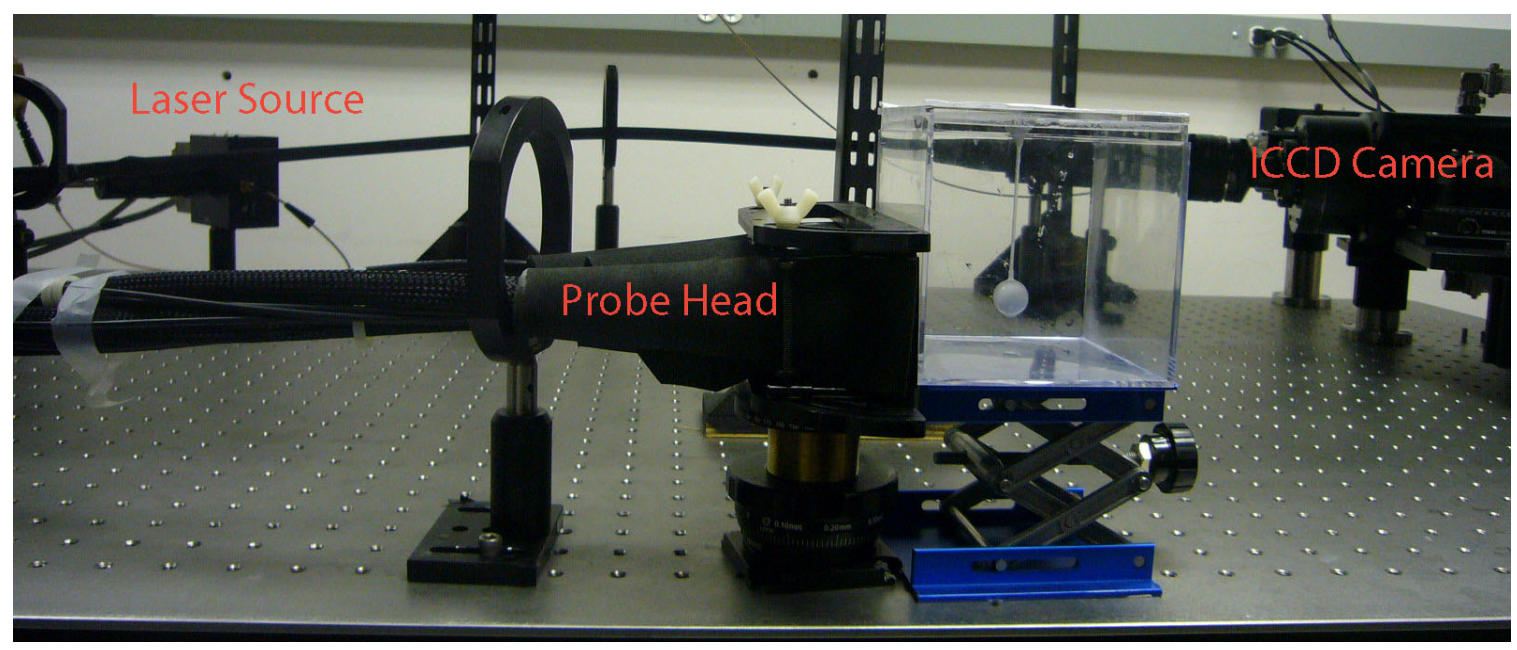

Figure 2.7 OIL's hand held optical imager and a blank phantom where (i) is the source, (ii) is the detector and (iii) is the hand held probe head in contact with a blank phantom [Jayachandran, et al. 2007]

Optical images collected by the optical imager (2D surfaces) are used in appropriate inversion algorithms to obtain 3D image reconstructions. However, 3D image reconstruction is only achieved if the probe's location is precisely tracked during imaging and superimposed with respect to the imaged surface. The recorded probe head position is acquired using a $3 \mathrm{D}$ motion tracker, which is attached to the probe head of the device. The accuracy of the position of a tumor within the tissue can be affected by the accuracy of the tracked position of the hand-held probe during imaging studies. Optical data collected from the camera is superimposed with the tracker data collected from the tracker in a process called co-registration (described in detail in Section 2.6). Therefore, for accurate $3 \mathrm{D}$ image reconstructions, an accurate positional tracker is required along with a post-processing tool to co-register for 3D tomography studies. In the following sections the approach of positional tracking and co-registration implemented for the current hand-held optical imaging system are described. 


\subsection{Tracking Devices}

A tracking device is a useful tool towards $3 \mathrm{D}$ reconstructions since it allows the probe head's location to be identified on a computerized mesh of the subject. Tracking devices that use mechanical, inertial, electromagnetic, acoustic, or optical methods can be used to track Optical Imaging Laboratory's (OIL) probe head [Regalado 2009]. However, mechanical trackers have limited mobility since the object must be affixed to a reference point. Inertial trackers, by nature tend to drift their measurements over time, affecting their accuracy. Therefore mechanical and inertial trackers have not been considered to track the probe head. Since probe head is required to be moved freely in order to obtain multiple images, a positional tracker such as electromagnetic, acoustic, or optical is desired.

Originally an acoustic tracking device from Logitech is in use with the hand held optical probe. The method of tracking used is composed of a transmitter to emit ultrasound waves towards a receiver within its field of view. The receiver has three microphones and is connected to a control box. A transmitter is also connected to the control box and emits the ultrasonic wave towards the receiver. Each microphone on the receiver obtains an acoustic signal that is used to determine its position in space. Figure 3 illustrates the orientation of the acoustic tracker and the setup of its hardware for proper functionality. The acoustic tracker is dependent on a field of view from the transmitter to the receiver, where figure 2.8 illustrates the range of vision as well. Although the acoustic tracker can be accurate, its drawbacks include the lack of flexibility to be customized for a smaller area and instability of the measurements, which can affect the accuracy drastically. The original tracker software was written in $\mathrm{C}$ programming 
language and its positional data obtained in real-time. However, in order to make the tracker functional with the LabView based co-registration software (see Section 2.6), the data obtained from the tracker was converted via MatLab to positional data appropriate for co-registration. The script converting the raw data to MatLab values causes the tracker to have a lag time (each time that the receiver is moved) of approximately 1 second.
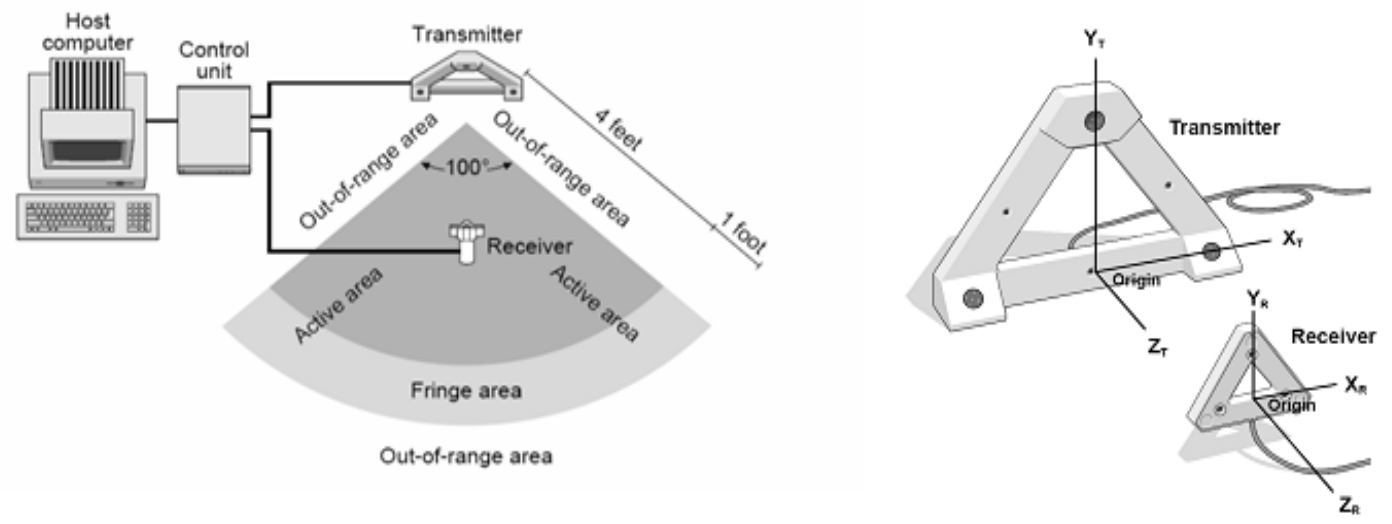

Figure 2.8 Acoustic Tracking Device (Logitech tracker manual, Logitech Inc., Fremont, CA.)

To improve upon the current method of tracking's instability, two other types of tracking methods will be analyzed, electromagnetic and optical. Table 2.1 describes the advantages and disadvantages of each of the trackers, which is described in more detail. Electromagnetic (EM) trackers are well known to be used in various applications (Raab, et al. 1979; Roetenberg, et al. 2007), are accurate, and do not require large hardware for tracking. The EM tracker uses orthogonal coils on the receiver and transmitter to determine the magnetic field vector of the coils. The relative magnetic field of the receiver and the transmitter determines the position and orientation of the receiver. They are very fast and very accurate even when obstructed by a large object since they do not 
require a field of view. However, if metallic objects are present near the magnetic field of the tracker, the data is greatly affected.

Optical trackers operate very similar to the acoustic tracker since they require a field of view in order to track the object and vary in prices depending on the components necessary. The optical tracker can be very fast and very accurate depending on the detector used (Meeks, et al. 2005; Reed 2002). The electromagnetic, acoustic, and optical trackers will be assessed for accuracy of tracking to improve the accuracy of the co-registration (see Chapter 3). 


\begin{tabular}{|l|c|l|}
\hline & Advantages & Disadvantages \\
\hline Acoustic Tracking & $\bullet$ Not affected by & $\bullet$ Requires line of \\
\hline
\end{tabular}




\begin{tabular}{|c|c|c|}
\hline & $\begin{array}{l}\text { metallic objects or } \\
\text { magnetic fields }\end{array}$ & $\begin{array}{l}\text { sight between } \\
\text { transmitter and } \\
\text { receiver } \\
\text { - Expensive } \\
\text { - Cannot be } \\
\text { customized } \\
\text { - Unstable } \\
\text { Lag time of } 1-2 \mathrm{sec}\end{array}$ \\
\hline $\begin{array}{l}\text { Electromagnetic } \\
\text { Tracking }\end{array}$ & $\begin{array}{l}\text { - No line of sight is } \\
\text { necessary } \\
\text { - Can be wireless } \\
\text { - Relatively Fast }\end{array}$ & $\begin{array}{l}\text { - } \text { Expensive } \\
\text { - Affected by metallic } \\
\text { objects and } \\
\text { magnetic fields }\end{array}$ \\
\hline Optical Tracking & $\begin{array}{l}\text { - } \text { Low cost } \\
\text { - } \text { Easily customized } \\
\text { - Wireless } \\
\text { - } \text { Fast and Stable }\end{array}$ & $\begin{array}{l}\text { Requires line of } \\
\text { sight between } \\
\text { detector and source }\end{array}$ \\
\hline
\end{tabular}

Table 2.1 Comparison of trackers towards tomographic imaging with OIL's hand held probe shows that optical tracking may provide to be the most effective.

\subsection{Co-registration with OIL's Optical Imager}

Co-registration is the method by which the collected image is super imposed with 
the positional data and then projected onto a computerized representation of the imaged surface [Regalado, et al. 2009]. To obtain a 3D reconstruction of a 2D image collected via an optical imager, the collected image must be superimposed with the positional data with respect to the imaged surface. The custom developed co-registration software (written in LabView) acquires the data collected from the optical imager (using WinView software) and superimposes it onto the tracker's positional data (obtained using MatLab) in order to obtain a co-registered image. Figure 2.9 illustrates the combination of the various software outputs to obtain a single co-registered image. The optical images acquired via Winview are initially saved and further uploaded into the LabView based co-registration software, making it a time consuming process. On the other hand, the tracked positional information in Matlab is an in-built part of the co-registration software, with least lag time $(\sim 1 \mathrm{sec})$. This multi-software based co-registration approach causes the entire process of obtaining a co-registered image as long as 5 minutes each time. When multiple images need to be acquired and co-registered towards 3D tomographic imaging, the entire imaging process might take minutes to hours. Hence, there is a need to enhance the efficiency of the co-registration software by reducing the overall image acquisition time. 


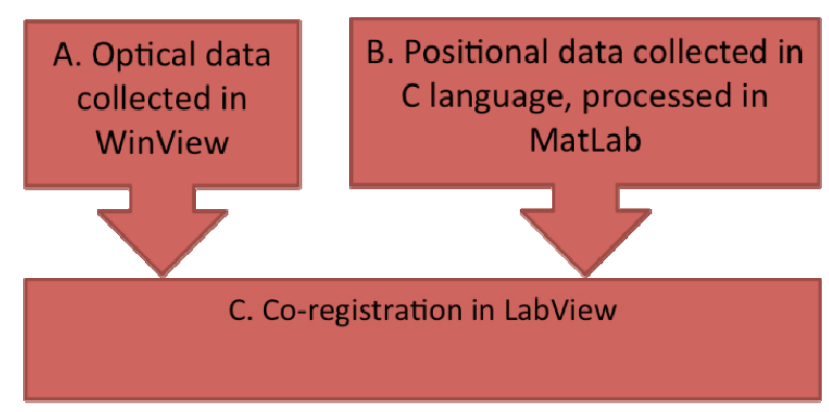

D. Co-registered image on a cubical phantom

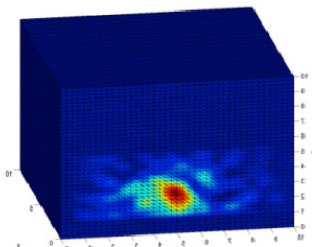

Figure 2.9 Graphical depiction of the co-registration software's function in (A) obtaining optical data from

WinView and (B) positional data from MatLab simultaneously. The data is then (C) super imposed in LabView to obtain the (D) co-registered image on a mesh of the surface.

\subsection{Summary}

In summary, the combination of the unique features of the developed optical imager (described in Section 2.4) has provided a novel method of 3D optical tomography via the use of a hand held probe head. Although all these features are unique and have provided the ability to perform 3D tomography, the hand held optical imager experiences the following shortcomings:

1. Lack of full contact with tissue: The three plate design of the probe head has sharp edges that do not make contact with the tissue.

2. Uneven source distribution: The six sources are collimated from a single laser where there is an uneven intensity distribution among the six sources. This uneven source distribution is difficult to account for in image reconstruction. 
3. Multiple software co-registration: Multiple software causes the imaging processing to be longer, thus increasing the overall imaging time in a clinical set-up.

4. Unstable positional tracking: Instability arising from the tracker affects the accuracy of the reconstructions through error in the positioning of the collected images.

The potential solutions to these shortcomings have led to the development of the next generation imager. These solutions include:

1. A more flexible probe head with smooth (and no sharp) edges to contour with maximum contact onto different tissue curvatures (part of another student's research in the laboratory).

2. An alternate instrumentation at the source end such that it provides uniform intensity of multiple simultaneous sources (part of another student's research in the laboratory).

3. A modified co-registration software that is fully-automated and also minimizes the overall co-registration time (my thesis focus).

4. An alternate tracking device with improved stability and accuracy, at a lower cost and smaller size (my thesis focus).

Herein, my research goals are primarily focused:

Specific Aim 1: To reduce the imaging time during co-registration by minimizing the amount of manual input necessary for the software to complete the image acquisition and co-registration process. In order to 
achieve a shorter time of completion, an automated software will be developed that will perform all the functions performed separately by Winview, MatLab and LabView.

Specific Aim 2: To improve the accuracy of the co-registered imaging via the use of efficient tracking devices. Electromagnetic, acoustic, and optical trackers will be assessed and compared. The comparison of the three trackers will determine the best fit for the optical imager.

My research goals will be primarily focused with improving the first generation imager. Once the first generation imager has shown improvement, the work completed will be implemented towards the second generation optical imager. 


\section{Chapter 3: Materials and Methods}

The multi-software co-registration approach used in the first generation optical imager [Ge, et al. 2008; Ge, et al. 2009] will be converted to an automated software package. As the co-registration software is automated with a new software approach, the time of imaging will improve. However, improving the time for imaging will not suffice unless the accuracy and stability of positional tracking are improved. By comparing multiple tracking approaches, a more accurate and stable device can be selected to improve the overall accuracy of co-registration. The following chapter describes (i) the methods of automating and improving the co-registration process and (ii) the method of improving the tracking accuracy for the first and second generation optical imagers.

\section{Part I Modifications Towards the First Generation Optical Imager}

\subsection{Automation of Co-registration}

The original co-registration software for the imager involved a multi-software (MatLab, WinView, and LabView) co-registration approach to achieve a co-registered image as described in Section 2.5. A complete automation of the co-registration and image collection process was ideal for reducing the time of co-registration. By automating the image acquisition process with the positional data acquisition and coregistration, the amount of time to collect and co-register one image can be reduced. A simple graphical user interface (GUI) was created along with the automation to minimize user input. Figure 3.1 below is the part of the GUI where the images are collected. A simple click of a button acquires the image and then can be co-registered in another part of the GUI. Figure 3.2 below shows the flowchart for the process of the automated co- 
registration software. The area labeled in A within the dotted box was originally the WinView based detector control and image acquisition software. The part labeled B, outside of the dotted box in the flowchart is the area which was created in LabView for the co-registration of the collected images. The flowchart describes the sequence of steps performed by the co-registration software. First the camera is initialized and focused, before an image is acquired (Steps 1-3). Parallely, the position of the hand-held probe is tracked using the tracker in 3D (Steps 6-7). Step 8 in the flowchart is the mathematical operation that correlates (superimposes) the positional data (Step 7) and the acquired image (Step 3) in order to position the 2D image on the (simulated) computerized probe. A post-processing subtraction technique is employed to account for background noise in the optical images during the image acquisition process (Step 9). This step is optional and used as needed during imaging studies. Prior to imaging studies, a discretized mesh of the tissue phantom being imaged is generated (Step 4) and uploaded into the coregistration software (Step 5). The correlated (and post-processed) optical images and positional data (from Step 9) are co-registered onto the 3D discretized tissue phantom mesh (Step 10) using the uploaded mesh (Step 5). The co-registered images are displayed (Step 11) and further stored (Step 12), such that the software is ready for acquiring the next set of images in near-real time until the experiment is complete (Step $13)$. 


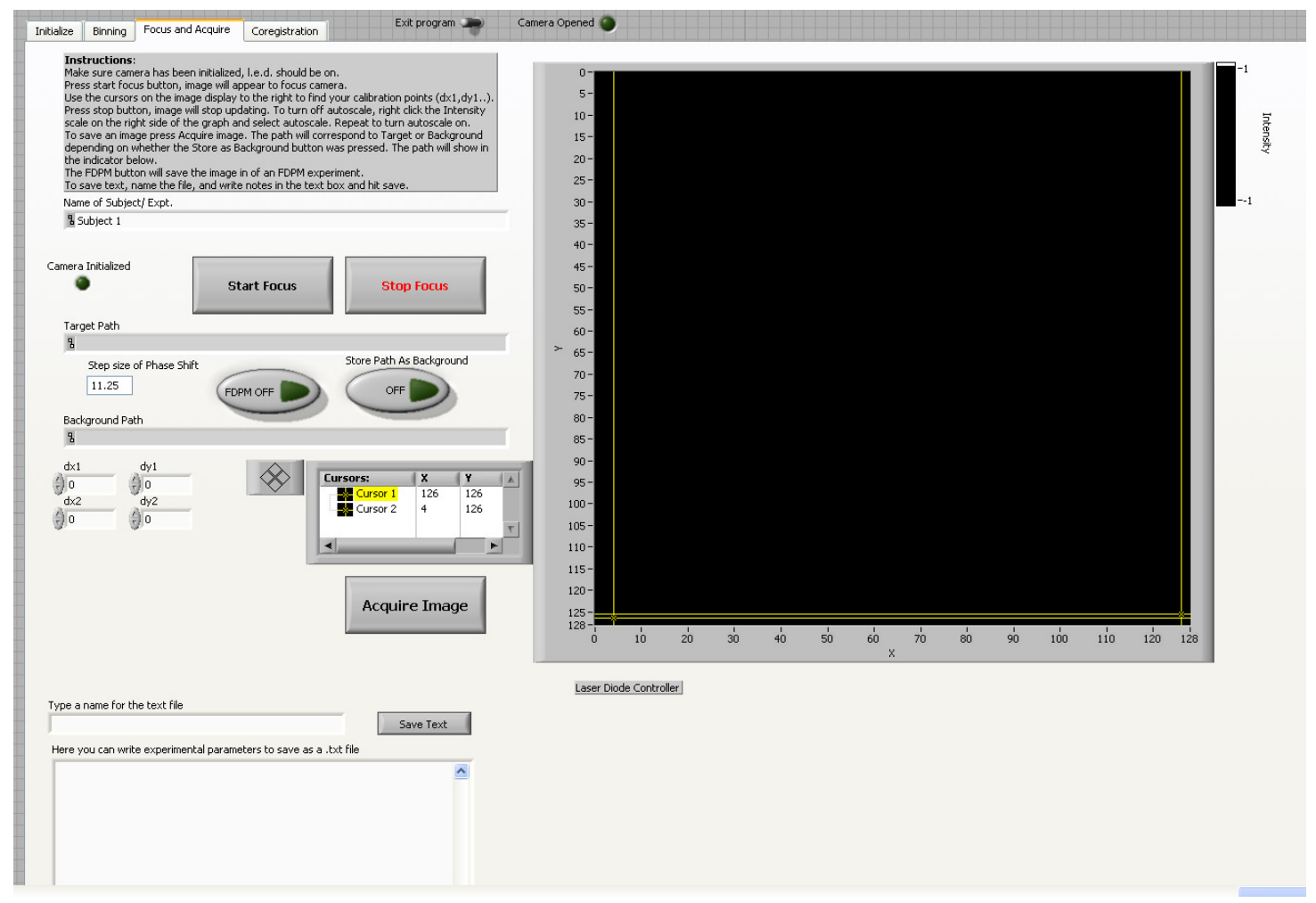

Figure 3.1 Graphical user interface of the new automated software. The focus window on the right shows the raw image from the camera. Once the "Acquire Image" button is pressed the $2 D$ image collected is displayed. 


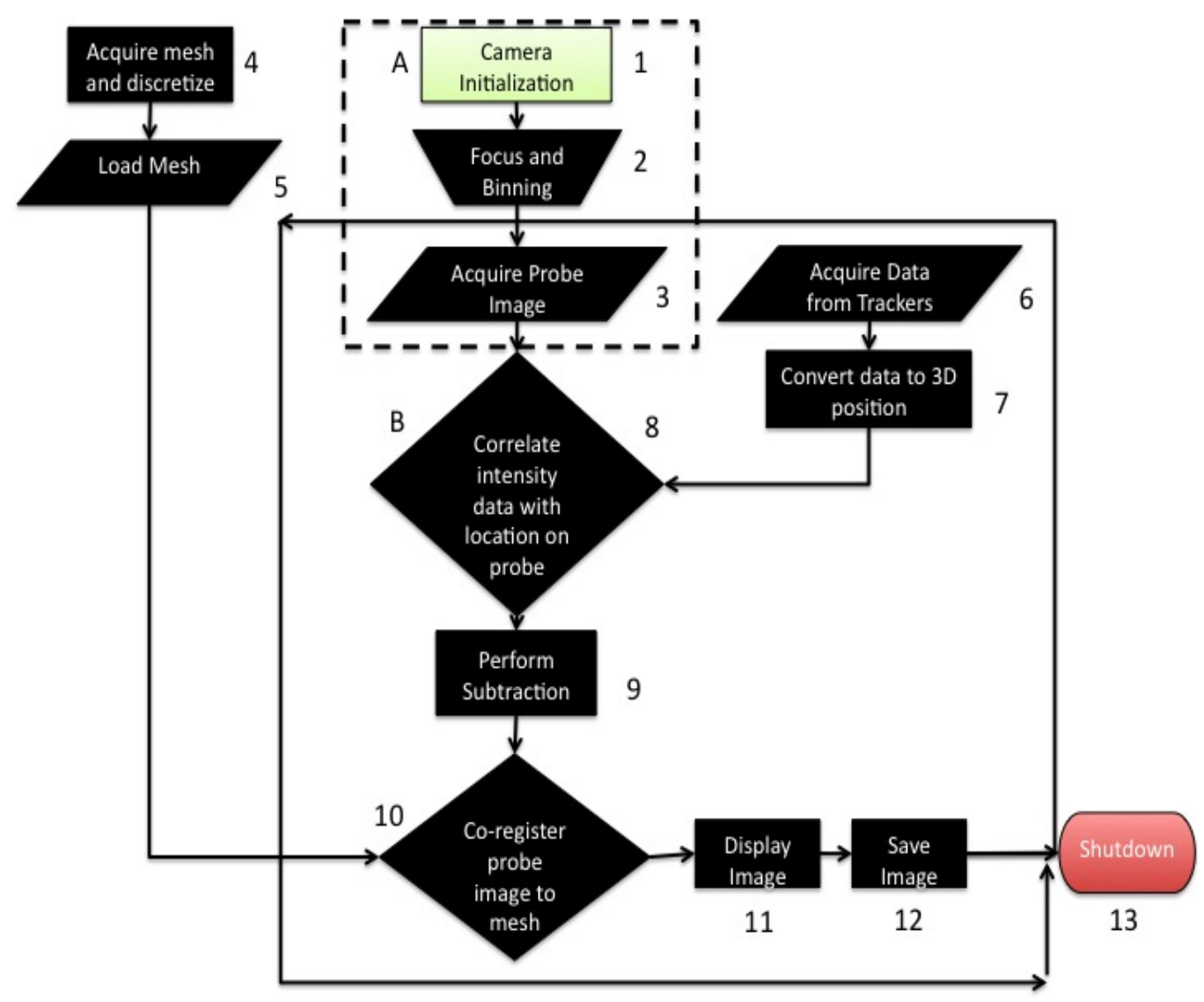

Figure 3.2 Flowchart of the software. (A) is where the previous software was run through WinView and has now been integrated with (B) the LabView based co-registration software for full automation.

\subsection{Study I: Experimental Evaluation of the Automated Software}

The automated software was tested for comparison of the time of one image collection with the multi-software approach. Comparison of imaging time was performed during phantom studies where image data collection was done using continuous wave measurements from tissue phantoms. For both software the same computer was used which has a dual core (virtual quad core) Intel $3.2 \mathrm{GHz}$ processor and 4GB of RAM on Windows XP (32-Bit). 


\subsection{Study 2: Tracker Evaluation}

Currently an ultrasound tracker is used with the hand held optical imager. However, it has been observed throughout our experiments that there is a significant amount of instability during the tracking process. A fluctuation of about $+/-.5 \mathrm{~cm}$ about the true position of the tracker has been observed. In order to reduce error, a more stable tracker is desired. Therefore, two different trackers were assessed, which include electromagnetic and optical trackers. For each tracking device, similar methods of accuracy testing were performed and will be described in the following Sections.

\subsubsection{Study I: Effectiveness of three trackers towards 2D positioning}

The effectiveness of the three tracking devices in accurate positional information in real-time was assessed via a comparison study. The effectiveness of the three trackers was assessed from 2D studies wherein the receiver or source end of the trackers was moved along the four corners of a phantom and the horizontal and vertical positional information was collected (keeping the depth constant). The measured positions and true physical positions were compared to determine the most effective tracker towards improvement of the co-registration for $2 \mathrm{D}$ co-registered imaging with the hand-held probe.

\subsubsection{Electromagnetic Tracker Analysis}

The study involved the evaluation of the electromagnetic tracker (Polhemus FasTrak, Polhemus, Colchester, VT) in comparison to the acoustic and optical trackers. The Polhemus Fastrak utilizes a wired setup where the receiver and transmitter are connected to a control box as shown in figure 3.3. Polhemus provides a stand-alone 
software to collect positional data from the EM tracker. To test the tracker, the receiver was placed on a single face of a blank cubical phantom. The square face of the phantom measures $8.5 \mathrm{~cm} \times 8.5 \mathrm{~cm}$ and the receiver end of the tracker was placed at each of the corners of the face (seen in figure 3.4) and measured physically as well as using the software. The accuracy of the tracker was determined by comparing the physically measured position of the receiver with the given position form the software. To test the accuracy of the magnetic tracker in true experimental situations, the tracker was also attached to the first generation hand held probe, which contains metal, in order to observe the effect that the metal has on its positional data.

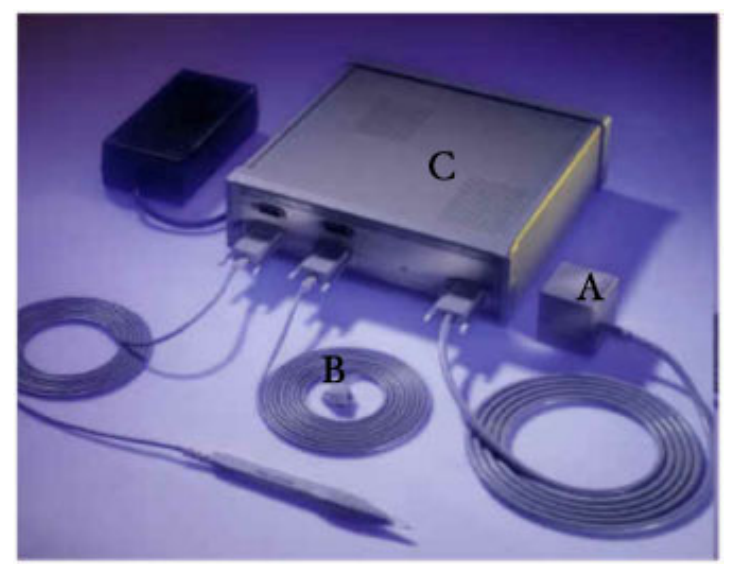

Figure 3.3 Polhemus Fastrak Electromagnetic Tracking Device. (A) is the transmitter, (B) is the receiver, and $(C)$ is the control box 


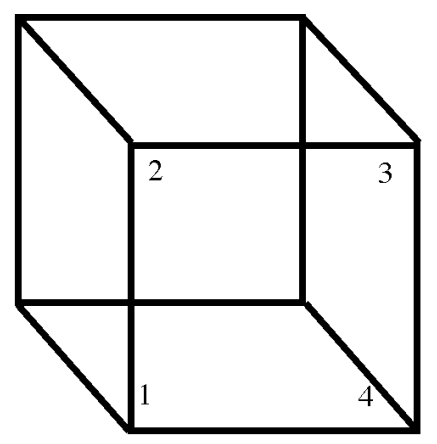

Figure 3.4 Representation of the cubical phantom with the labeled points of where the tracker receiver was placed

\subsubsection{Acoustic Tracker Analysis}

The study also involved an evaluation of the acoustic tracker (Logitech 3D Head Tracker in figure 3.5) for comparison with the EM and optical tracking devices. The method of assessing the tracker's accuracy was similar to the EM tracker where the receiver was placed at each of the corners of a single face of a blank cubical phantom and measured physically as well as through the software. The accuracy was compared through individual point collection and continuous data streaming, which was obtained for the determination of stability while moving the receiver, as well as leaving the receiver at rest.

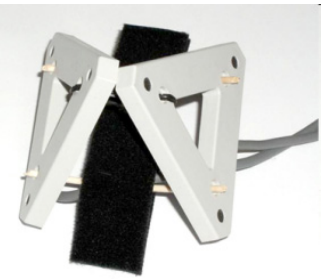

Figure 3.5 Logitech Ultrasound 3D Head Tracker 


\subsubsection{Optical Tracker Analysis}

The optical tracker (which will be discussed in section 3.4) was a customized Nintendo Wii Remote and was compared the EM and acoustic tracker. The method of assessing the tracker's accuracy was similar to the EM tracker where the receiver was placed at each of the corners of a single face of a blank cubical phantom and and measured physically as well as through the software. The accuracy was compared through individual point collection, which was measured physically as well as through the custom software. Continuous data streaming was also obtained for the determination of stability while moving the LEDs, as well as leaving the LEDs at rest.

\subsection{Optical Tracker Customization}

The optical tracker chosen for the current study was a very inexpensive tracker originally used as a controller for a video game console. A Nintendo Wii Remote, or also called the Nintendo Wiimote (see figure 3.6), was used as the optical tracker with an AZiO micro Bluetooth Adapter for the computer connection. The Nintendo Wiimote

contains an infrared camera with a $1024 \mathrm{X} 768$ pixel resolution with built-in post processing allowing for four LEDs to be tracked at a time. 


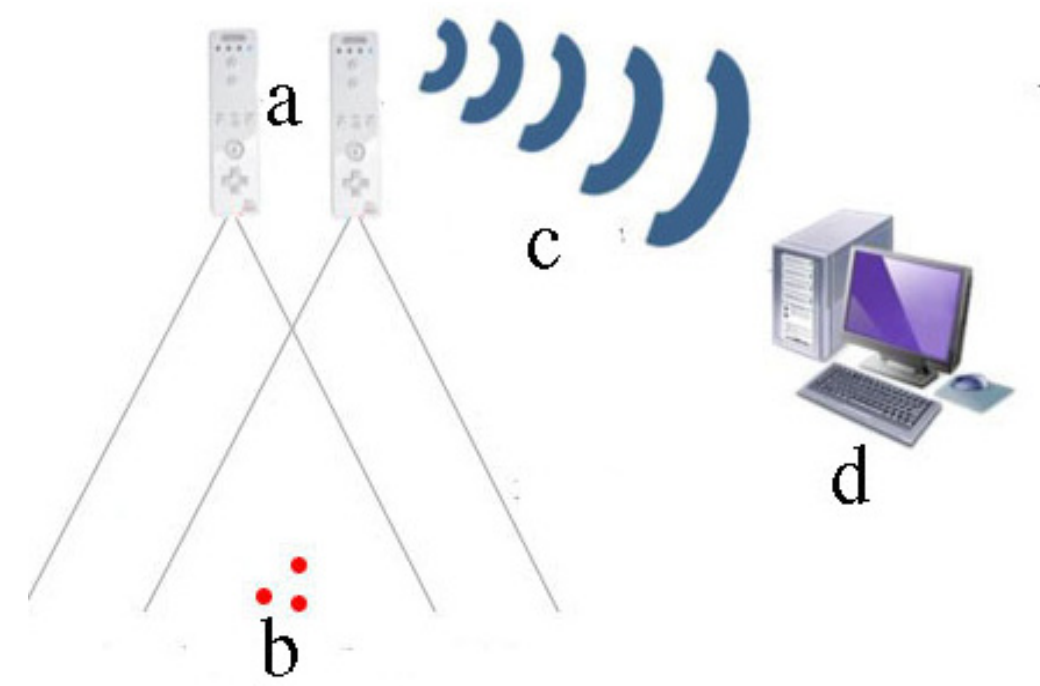

Figure 3.6 Nintendo Wii controllers used as a tracking device where (a) are the Wii controllers, (b) are the tracked infrared LED's, and (c) is the Bluetooth waves from the Wii controllers that allow wireless communication with (d) the host computer.

In order for the tracker to be used with a computer, a connection must be made through Bluetooth and a custom code written to use its tracking capabilities. There are very few Bluetooth software, or Bluetooth stacks, which can appropriately control the amount of information coming from the Wiimote as a tracking device. Therefore BlueSoleil was used to handle the Bluetooth communication between the computer and the Wiimote. The figure below shows the flowchart of the Wiimote tracking process. 


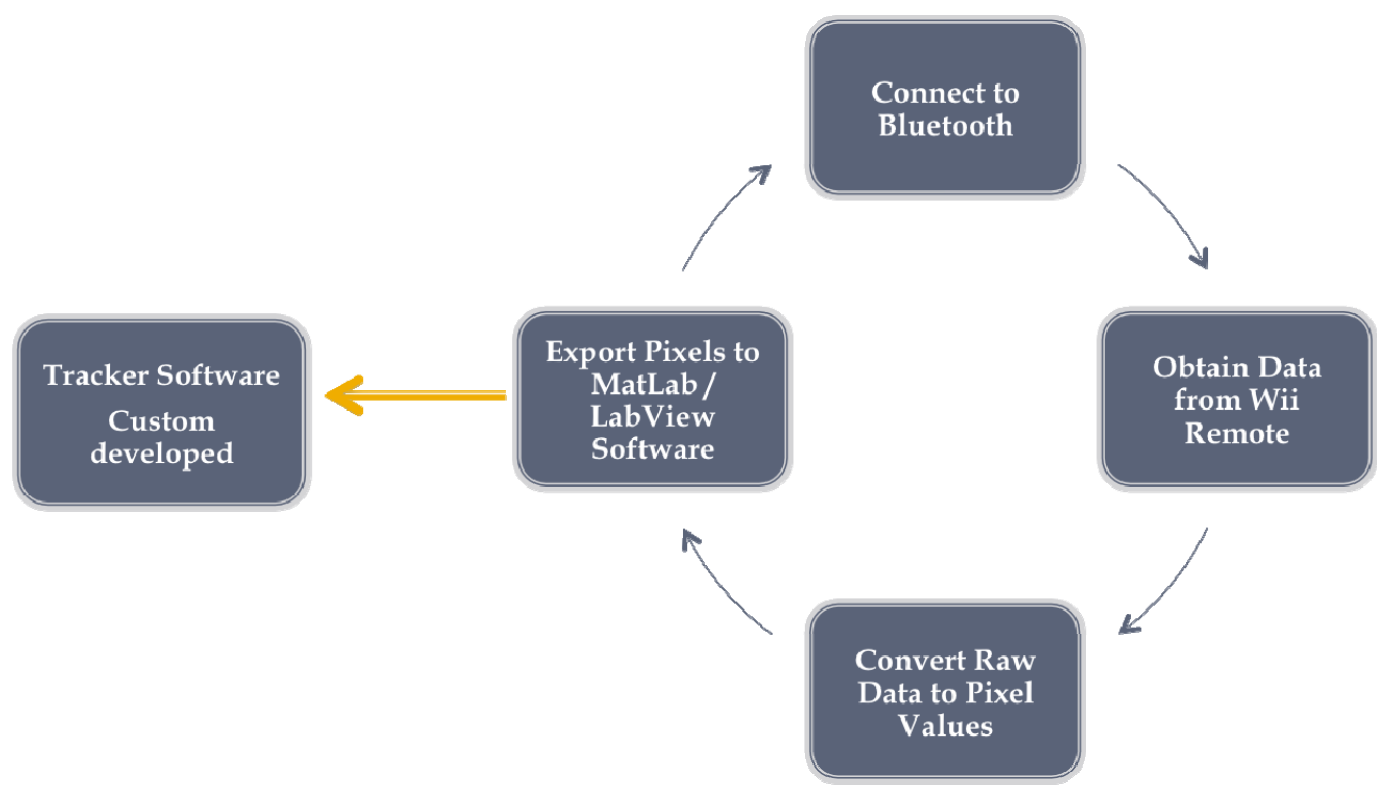

Figure 3.7 Flowchart of Wiimote Data Collection Process

\subsubsection{D Tracking LED Circuit}

The Nintendo Wiimote originally uses (infrared) LEDs for the Nintendo Wii's software to determine the position and movements of a user. Therefore, to use the Wiimote as a tracking device on a computer an infrared source array was developed to use as the object to be tracked. Infrared LEDs operating at $940 \mathrm{~nm}$ wavelength with a $100 \mathrm{~mA}$ forward current were used. Each circuit required 2 LEDs, three $1 \mathrm{Ohm}$ resistors per LED, and a $1.5 \mathrm{~V}$ AA battery as per Ohm's Law. The two LED's were placed in parallel in order to supply enough current to them from a single battery. Figure 3.8 below illustrates the circuit used. Since the LED array is the object to be tracked by the Wiimote, any object attached to the LED array will also be tracked. 


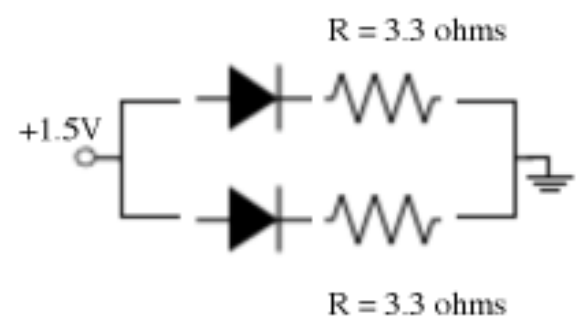

Figure 3.8 LED Circuit

\subsubsection{Converting Wiimote Raw Data to Distances}

The Wiimote was customized as required for the development of 2D tracking software since it was not inherently a tracking device. Tracking information acquired from the Wiimote is represented directly as raw data, where each given value by the Wiimote corresponds to a particular pixel value on the camera of the Wiimote. Therefore an experimentally derived equation was determined such that each pixel of the camera would correspond to a particular distance traveled in horizontal and vertical directions dependant on the distance the source was from the camera. To determine the equation for the conversion of the pixel value given by the camera to distance travelled by the LEDs, the Wiimote was placed at a fixed location and the source LEDs were moved. The LEDs were placed at 5 experimentally varied depths and 10 lateral/vertical positions at each depth, where each position was physically measured as shown in figure 3.9. Once the pixel value for each position and at each distance was given, equation 1 was determined through curve fitting. A sample data set collected at $30 \mathrm{~cm}$ depth is shown, where the formulae given are the best-fit line of the data collected. In order to achieve the actual slope of the line necessary for conversion as in equation 1, the slope of each line and for each data set was divided by the distance from which it was collected. An average was 
then taken for the slope of each data set and used as the conversion factor in equation 1. In order to test the accuracy of the determined equation, 30 known and measured positions were taken and the $\left(N_{x}, N_{y}\right)$ values given by the Wiimote were compared to the measured positions.

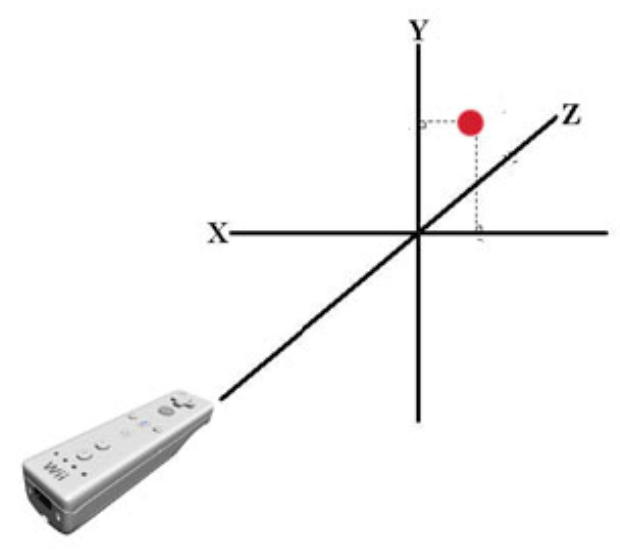

Figure 3.9 Setup for the 2D tracking equation. The Wiimote was placed at multiple distances from the $(x, y)$ plane where the LED was placed. The LED was then moved in the $x$ and $y$ direction to determine the equation to convert the pixel values to distances. 


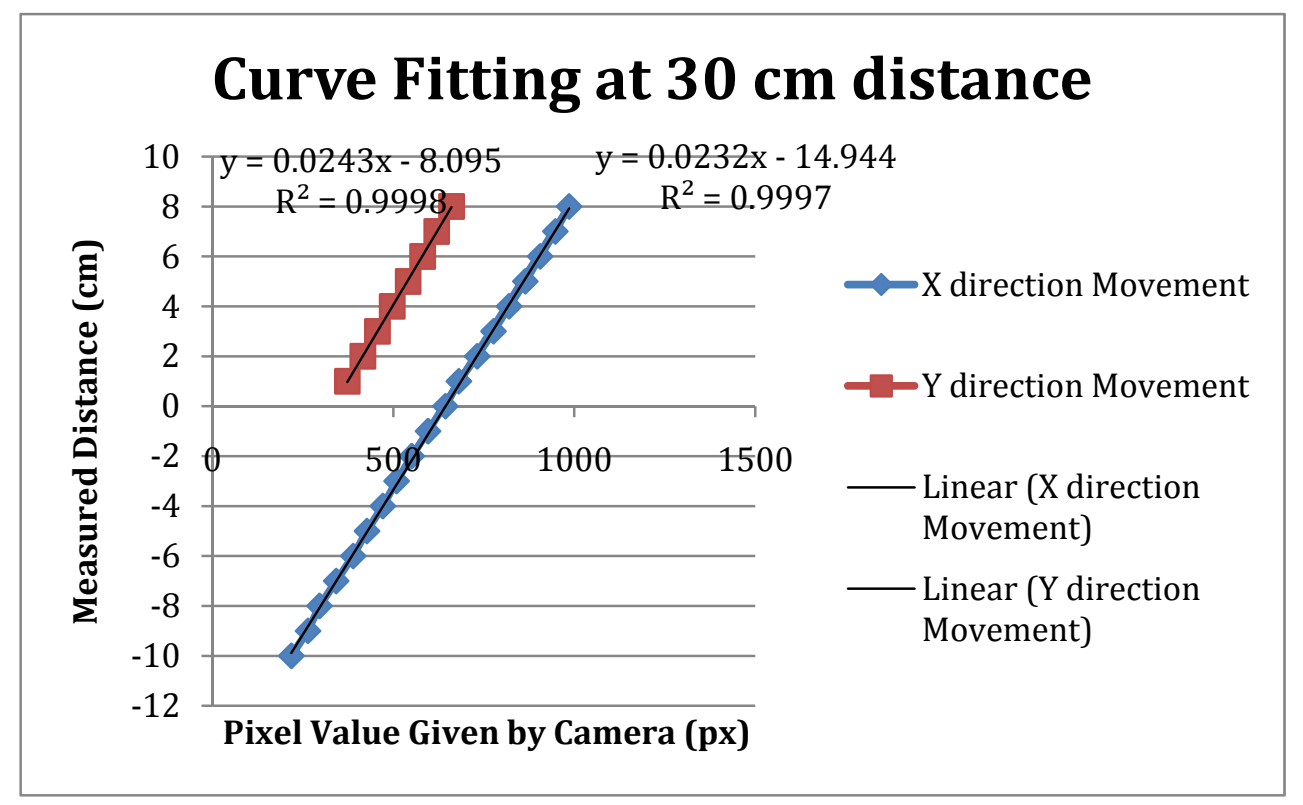

Figure 3.10 Sample of one data set of collected positions to determine the formula to convert pixels to $\mathrm{cm}$. As can be seen the $R^{2}$ value average for all samples collected was .99982 with standard deviation of $8.32 E$ 05. The plots show a linear relation between the measured LED position and the pixel value given by the camera. The black line represents the line of best fit.

$\left(N_{x}, N_{y}\right)=(.000783 \times d) \bullet\left(P_{x}, P_{y}\right)$ Equation 1

The variable $d$ in equation 1 represents the measured distance in centimeters that the source (i.e. LED) is from the detector (i.e. Wiimote), $\left(P_{x}, P_{y}\right)$ is the respective horizontal and vertical pixel value given by the detector (Wiimote), and finally $\left(N_{x}, N_{y}\right)$ is the actual position of the source (LED) in centimeters. At each distance, a line of best fit was determined for the data collected, where the distance measured for the position of the pixel was a function of the pixel value given by the Wiimote (as shown in Figure 3.10). Each data set had a plot similar to Figure 3.10 with the line of best fit given by the equation of a line, $\mathrm{y}=\mathrm{mx}+\mathrm{b}$. In order to determine the slope of the conversion equation (equation 1), the slope of each line was divided by the distance (between the Wiimote and 
LED) for each data set and then averaged. The value .000783 was determined to be the average slope (or the conversion factor) by the above formulation, with a standard deviation of 1.79E-05. The constant ' $b$ ' in this linear equation, $y=m x+b$, was not given in equation 1 , since it is given by what pixel value the user determines to be zero, and will be described in 3.4.4 (which is related to the calibration of the tracker). By using equation 1, the 2D location of the LEDs can be obtained as the LEDs are moved in real time, as long as the distance from the $x-y$ plane (where the LEDs are located) to the Wiimote is known (i.e. $d$ in equation 1).

\subsubsection{Operational Limitations of the Wiimote}

Once equation 1 was verified, the limitations of operation of the Wiimote were determined. Knowing the operating limits of the Wiimote, experimental setups can then be determined so that the LEDs are always in view of the Wiimote, preventing errors due to exiting the field of view or detection limits of the Wiimote. The maximum distance of detection of the LEDs was determined by moving the LEDs away from the Wiimote until the LEDs were not visible to the Wiimote, where the distance was found to be $173 \mathrm{~cm}$. Also, the maximal field of view was determined by fixing the Wiimote on an elevated surface and moving the IR LEDs known distances up, down, left, and right to detect the full field of view of the camera where the measured field of view was determined through simple geometry. Since the distance of the LED to the Wiimote was known, and the height of the LED was known, the angle at which the LED was from the Wiimote $(\theta)$ was determined as depicted in figure 3.11 and using equation 2. The maximum field of view angle of the Wiimote was found to be $43^{\circ}$. 


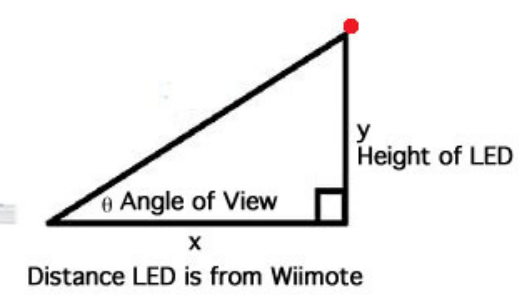

Figure 3.11 The angle for field of view was measured using simple geometry.

$$
\theta=\tan ^{-1}\left(\frac{y}{x}\right)
$$

Equation 2

\subsubsection{Optical Tracker 2D Integration to Automated Software}

In order to be able to perform the co-registration in an experimentally comparable method, the software developed for 2D tracking was integrated into the automated software. The acoustic tracker files were removed from the software preventing the software from attempting to communicate with a non-existent tracker. Once the files were removed, the files created for communication with the tracker were integrated in place of the acoustic tracker's files. The formula was directly encoded into the LabView software to quickly perform the calculations. MatLab was then used for the conversion of the Wiimote data in equation 1 and the output numbers for the LED location were used as inputs for the m-files that perform the co-registration mathematics. An input was added to the front panel of the software for the distance at which the LEDs are from the Wiimote, allowing for the calculation of the 2D position of the LEDs attached to the probe head. All the tracker data is collected and converted in real time to allow for the most accurate and fastest data collection possible. 
Figure 3.12 below describes the flow of the co-registration process used in the developed software. The co-registration begins by loading the 3D mesh of the phantom into the software and the communication with one or more Wiimotes is initiated. Communication with the Wiimote's allows for the real time acquisition of data from the Wiimote, which is converted to useful distance measurements through a MatLab script involving equation 1. The MatLab script is integrated to the Labview software to allow for automatic transfer of data through the software for real time tracking of the probe heads. In figure 3.12, the boxed area identifies the portion modified from the original ultrasound based co-registration software to the new optical tracking based coregistration software. The acquired images are co-registered onto the imaged surface by using the $2 \mathrm{D}$ positional information. Finally the image is saved and the process is repeated for each collected co-registered image. 


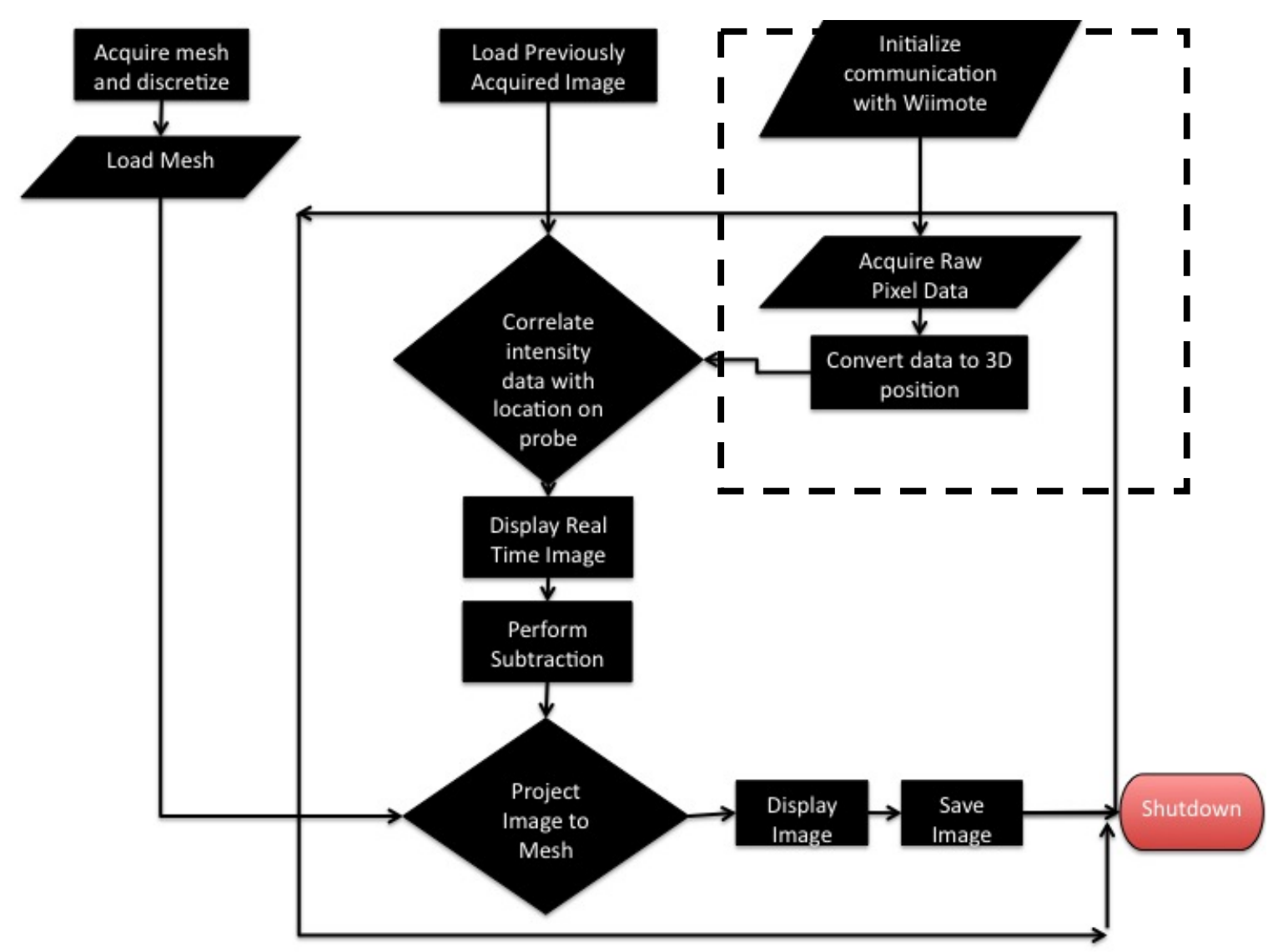

Figure 3.12 Flowchart for Wiimote Tracking Integrated into the Co-registration Software

Although the tracking system accurately converts pixel data to positional values, a reference point can be used in order to have a zero point and track all positions in relation to that reference point. Initially starting the tracker at a reference point will display an arbitrary value for the position of the LED. However, this initial position is the reference point, which requires a value of $(0,0)$. In order to reinitialize the system to zero at the reference point and determine all other positions in relation to the reference point, a method of re-initialization was developed. Subtraction of the initial position from every position given by the tracker allows the real positions and distances traveled to be displayed in relation to the reference point $(0,0)$ as given by Equation 3 . 
$\left(C_{x}, C_{y}\right)=\left(N_{x}, N_{y}\right)-\left(N_{o}, N_{o}\right)$ in cm

Equation 3

In equation $3,\left(C_{x}, C_{y}\right)$ is the new reinitialized position given by subtracting the initial $\left(\mathrm{N}_{0}, \mathrm{~N}_{0}\right)$ position of the tracker and the current $\left(\mathrm{N}_{\mathrm{x}}, \mathrm{N}_{\mathrm{y}}\right)$ position from the tracker. $\left(\mathrm{N}_{0}, \mathrm{~N}_{0}\right)$ can now be considered the ' $b$ ' (or $y$-intercept) of the line equation, $y=m x+b$, as described in Section 3.4.2.

\subsubsection{D Co-registration Testing with Tracker}

Implementation of the tracker was the first step of integration towards the first generation imager. Once the software was ready to be tested, the circuit developed for 2D tracking was used on an aluminum block to view the accuracy that the co-registration had of imaging (using a dummy image). A false image was loaded into the coregistration software, and a mesh of the cubical phantom was used. The aluminum block to be tracked was initialized at the bottom right of the cubical phantom and the false image was co-registered in position. The aluminum block was then moved in a manner mimicking the probe head's movements during a phantom study. The Wiimote was placed a distance of 25,35 , and $45 \mathrm{~cm}$ from the LEDs and the aluminum block was moved to 20 different $(\mathrm{x}, \mathrm{y})$ positions for each distance on a $20 \mathrm{~cm} \times 20 \mathrm{~cm} \times 20 \mathrm{~cm}$ cubical phantom. The accuracy of the tracker was determined, while testing the functionality of the co-registration software. Figure 3.13 below shows the set up of the study that was performed to determine the accuracy of the optical tracker during co-registration of a previously recorded image without the use of the probe heads. The phantom was annotated (using tape in two axes) to easily read the measured positions on the face of the phantom on which the LED source were to be placed and to compare the results given 
from the computer to those measured positions. The co-registered images were saved and the values given by the co-registration software regarding the positions were compared to the physically measured values.

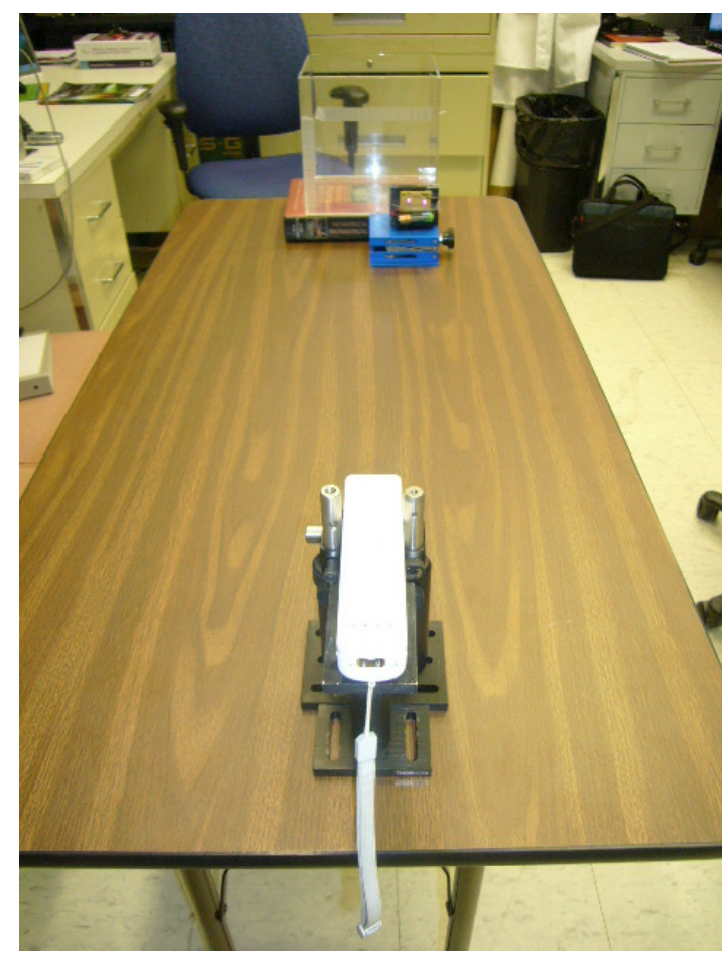

Figure 3.13 Setup for Tracker and Co-Registration Testing

\subsubsection{Optical and Acoustic Tracker 2D Co-registration with Probe}

The optical and acoustic trackers were compared in their ability to track the probe head as well as their impact on the position of the target in a phantom. As explained in section 3.3, the position of the receiver corresponds to the position of the probe head. The receiver of the acoustic tracker and the LED's of the optical tracker were placed on the probe head and used with the co-registration software during phantom studies. A large cubical phantom $(20 \mathrm{~cm} \times 20 \mathrm{~cm} \times 20 \mathrm{~cm})$ was filled with $2 \%$ Liposyn solution. A target with $1 \mu \mathrm{M}$ indocyanine green (ICG) of size $0.45 \mathrm{cc}$ was placed in the phantom at 1 
cm depth, $5.5 \mathrm{~cm}$ vertically, and $9 \mathrm{~cm}$ horizontally as figure 3.14 depicts. The probe head was placed directly in contact with the face of the phantom and images were collected at heights of 1,2, and $3 \mathrm{~cm}$ vertically and 4, 5, and $6 \mathrm{~cm}$ horizontally for a total of 9 images for acoustic and optical tracker based hand-held probe. At these multiple positions the target is visible in the images collected and the positions can be compared to the physical position of the probe head. In addition to $2 \mathrm{D}$ and $3 \mathrm{D}$ location of the probe head, the images collected are also used to determine the effect the tracking device has on locating the position of the target. The target is in a known location and the images that are collected will provide the coordinates for which the target is found on the probe face.

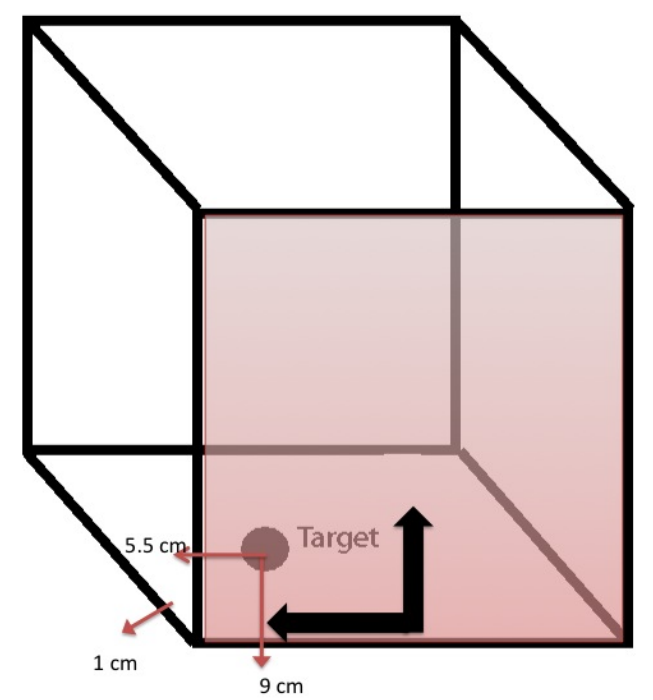

Figure 3.14 The figure depicts the motion that was performed on the large 20x20x20 cm phantom with the probe head and tracker attached to obtain the location of the target in the images collected. A $2 \%$ Liposyn phantom and a $1 \mu M$ indocyanine green (ICG) .45cc target were used. The arrows show the direction of the of the movements of the probe head. 


\subsubsection{Fine Movement Tracking with Optical Tracker}

The optical tracker was also tested to determine the capability of tracking down to $1 \mathrm{~mm}$ movements. The capability of tracking down to only $1 \mathrm{~mm}$ movements was tested and now below that value since the limitations of the human hand movement during imaging (using the hand-held probe) in less than $1 \mathrm{~mm}$ increments. In other words, the smallest increments that would ever be attempted using our hand-held probe is $1 \mathrm{~mm}$ increments, and hence the capabilities of the Wiimote tracking at these small increments is tested. An infrared LED was placed on a block and moved from 0 to $2 \mathrm{~cm}$ in $1 \mathrm{~mm}$ increments with the Wiimote placed a distance of $40 \mathrm{~cm}$ from the LED. The study was repeated 3 times and the accuracy of the position at each $1 \mathrm{~mm}$ increment was compared to the real position of the LED. After determining the position and averaging the error $1 \mathrm{~mm}$ movements were tracked with a $7.31 \%$ error and a standard deviation of .0818 .

\subsection{Customization of Optical Tracker for 3D Tracking}

The determination of the position of an LED in 3D space requires basic triangulation method. The formula's used for triangulation are based on the position of the two optical trackers and the angles determined by the tracker. The information regarding the 3D tracking can be found in Appendix 1 "Three Dimensional Tracking."

\subsection{Optical Tracker LED Affect on Imaging}

In addition to accuracy testing, it is necessary to identify if the infrared LEDs used for tracking will have an effect on the images collected. Since the camera of the imager collects infrared signals, the camera may collect a signal from the LEDs operating at $940 \mathrm{~nm}$ wavelength. In order to test if the LEDs affect the signal collected by the 
camera, images were collected with a phantom and active LEDs on the probe head to determine if a signal was collected from the LEDs. The results collected from these experiments were assessed. In the following chapter the results will be described in detail.

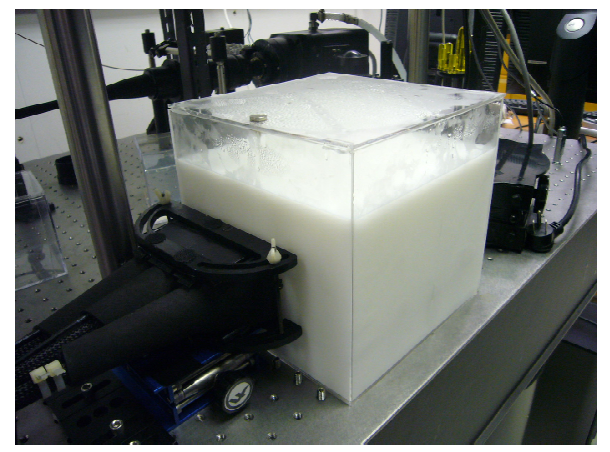

LED off

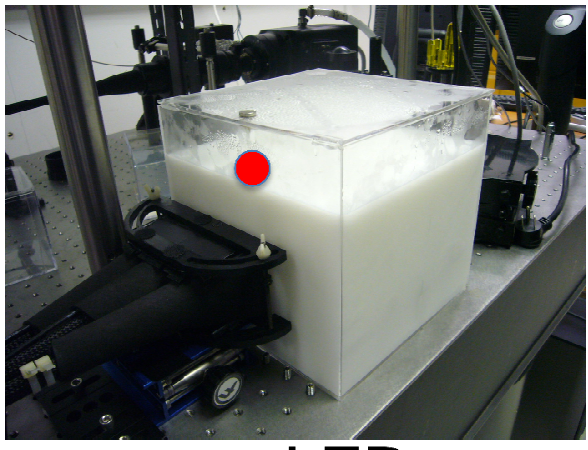

LED on

Figure 3.15 Depiction of how the effect of the LED on imaging was performed where a 2\% Liposyn phantom was used and the LED was placed on the surface of the phantom and on the center of the probe head to see if the IR signal from the LED effects the images collected.

\section{Part II Modifications Towards Second Generation Optical Imager}

\subsection{Second Generation Optical Imaging Device}

The first generation optical imager has been proven effective and used for phantom, in vitro, and in vivo studies. However, through the use of the optical imager, many areas for improvement were found including a more flexible probe head for maximum contact and an improved source distribution among others. Through the design of a second-generation probe head, a full second-generation system was developed with advantages over first generation as described in table 2.1.

Firstly, the probe face was manufactured from a single silicone based material allowing for it's inherent curvature to match any curved surface and provide maximum 
contact with the breast tissue. The probe head was developed as two smaller area probe heads that allow for reflectance and/or transillumination measurements to be collected. The number of sources remains the same (6), although the number of detector fibers was increased from 165 to 192 . This helped cover the entire area of the two probe heads of 5 $\times 4 \mathrm{~cm}^{2}$ each with $0.5 \mathrm{~cm}$ spacing between detector fibers. Figure 3.16 shows the difference between the first and second generation probe heads. The design and development of the second-generation probe was carried out by a fellow graduate student in the laboratory.
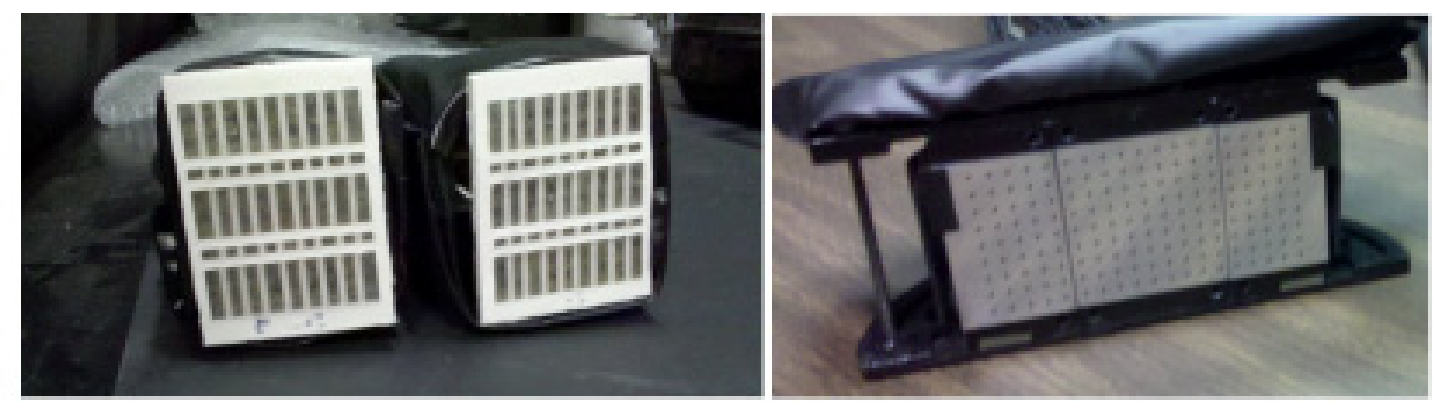

Figure 3.16 Second Gen Probe Head (left) and the first generation probe head (right). Although the second generation probe heads may be smaller individually, they have more flexibility and are one flexible piece on the probe face allowing for maximum contact while the first generation optical imager is three individual solid plates.

The probe head was one of many improvements to the optical imager, however the source power distribution had to be more evenly distributed. The first generation optical imager had one laser diode attached to a collimator package to split the laser into 6 ideally equal power point sources. Ideally a collimation package would distribute the power between the six sources equally, however, that was not the case. The source distribution of the first generation imager had uneven source strengths across the 6 
sources. Uneven distribution of sources leads to potential failure in detection of tumors closer to weaker sources compared to the stronger sources. The second-generation optical imager uses individual laser diodes for each of the six source fibers. They are all given the appropriate amount of power to give a maximum of $2 \mathrm{~mW}$ output from each source. By obtaining the appropriate amount of power at each of the six sources, the probability of missing a target due to a weak source is diminished.

In addition to improving the performance of the imaging system, a more compact design was desired in order to make the entire system portable. A cart was purchased and new equipment purchased and assembled to contain the camera, sources, and all other hardware necessary for the imaging system to function. An opening on the front side of the cart allows for the fibers of the hand held probe to travel out of the cart and gives maximum mobility of the hand held probes for imaging as seen in figure 3.17. Table 3.1 gives a brief comparison of the first generation optical imager and second generation optical imager with respect to the probe head design, imaging systems, and the coregistration approach (to be discussed in more detail in Sections 3.8 and 3.9). 


\begin{tabular}{|c|c|}
\hline First Generation & Second Generation \\
\hline \multicolumn{2}{|c|}{ Probe Heads } \\
\hline - $\quad$ Single probe head & - $\quad$ Two probe heads \\
\hline - $\quad$ Three plate probe face & - $\quad$ Single flexible face \\
\hline - Metallic probe face & - $\quad$ Silicon based material \\
\hline - 165 Detectors & - $\quad 192$ Detectors (96 per probe) \\
\hline \multicolumn{2}{|c|}{ Imaging System } \\
\hline - Immobile bench top & - $\quad$ Portable cart \\
\hline - 6 sources from one diode using custom- & - 6 source fibers, each with its own diode \\
\hline built collimator-diffuser package & ( 3 per probe head) \\
\hline - $\quad$ Liquid cooled CCD camera & - $\quad$ Air cooled CCD camera \\
\hline \multicolumn{2}{|c|}{ Co-Registration Approach } \\
\hline $\begin{array}{l}\text { - Single probe head co-registered to single } \\
\text { phantom }\end{array}$ & $\begin{array}{l}\text { - } \quad \text { Both probe heads co-registered to same or } \\
\text { separate mesh(es) of phantom(s) }\end{array}$ \\
\hline $\begin{array}{l}\text { - Unstable acoustic 3D tracking with } \\
\text { alternate optical 2D tracking }\end{array}$ & - $\quad$ Stable optical 2D multiple object tracking \\
\hline $\begin{array}{l}\text { - Subtraction only from target and } \\
\text { background images }\end{array}$ & $\begin{array}{l}\text { - Subtraction from target and background } \\
\text { image as well as between probe heads }\end{array}$ \\
\hline
\end{tabular}

Table 3.1 The comparison of features between first and second-generation optical imagers 

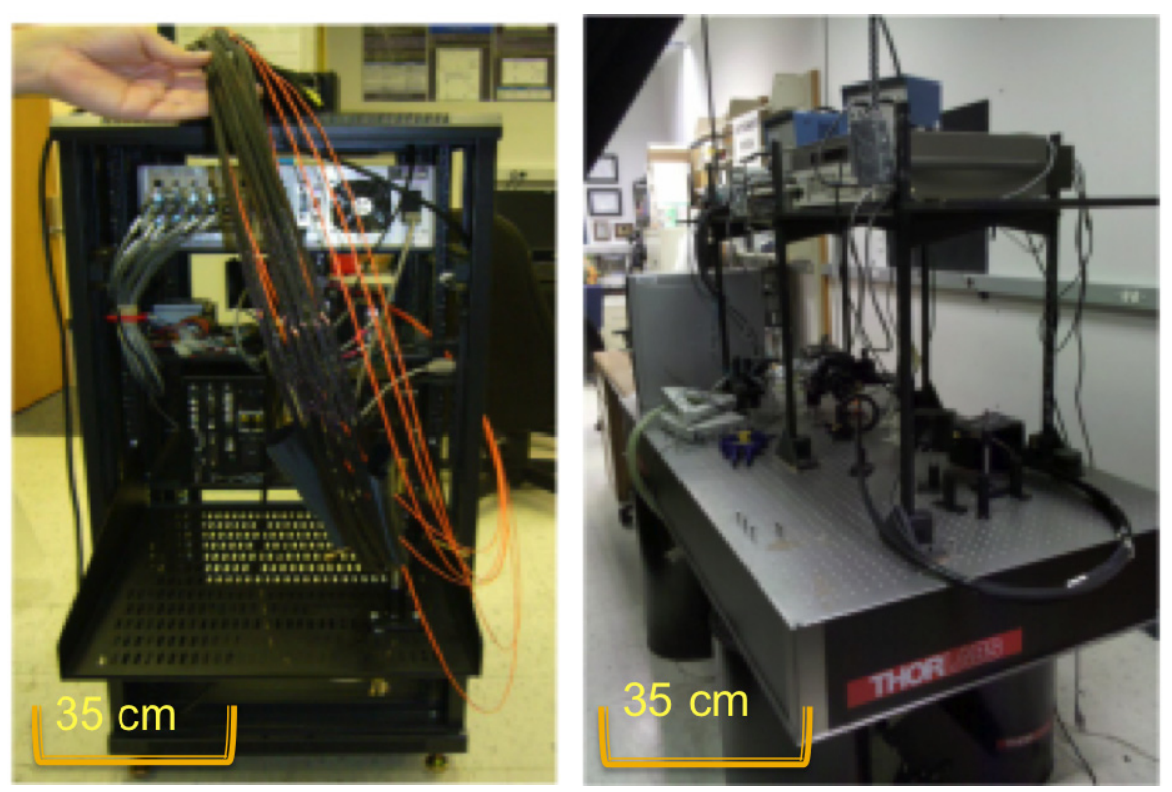

Figure 3.17 Second generation System in its Cart (left) compared to the first generation optical imager (right). It can easily be seen that the second-generation system is much more compact and portable than the bench top first generation optical imager.

\subsection{Software Development}

Since a new optical imaging system was developed and assembled for the second generation probe, an automated software package similar to the first generation system was necessary. The software developed for the first generation system was modified to control the temperature, gain, and laser powers for the second-generation imaging system. The camera used for the second generation system is a Roper Scientific PI-Max 2 intensified CCD camera. Similarly to the first generation system, the temperature and camera settings are computer controlled. However, the second generation system benefits from electronically controlled gain versus the manually controlled gain of the first generation imager. 
In addition to changing the controls for the camera, a new probe design was implemented. Therefore, the circular mount that holds the fibers and attaches to the camera is significantly different from the first generation probe head. The second generation probe head utilizes more fibers, which are arranged in a manner where half of the circular mount corresponds to one probe and the other half corresponds to the other. Thus a new code for converting the raw images of the plate to the $2 \mathrm{D}$ contour plots was developed. The original code to convert the raw data to the $2 \mathrm{D}$ contour plots that are later co-registered to the 3D mesh was modified for the second generation circular mount. The modified code was implemented into the automated software through a MatLab script node in LabView. All camera inputs are automatically input to the MatLab script node in LabView to allow for the fastest method of converting raw data from the camera to useful information. Prior to completing the entire second generation system, the second generation probe was used along with the hardware (ICCD and computer) from the first generation imager.

The multichannel controller used for the six individual sources of the second generation imager came with a LabView based software for automated control of the laser diodes. The multichannel controller software was modified and integrated to the automated software such that one or more laser diodes can be turned on or off remotely at the same time. All the modifications in the automated software (e.g. laser control, two probe head co-registration, etc.) lead to the evolution of the second generation automated software appropriate for the second generation imaging system. The modifications allow for simultaneous camera and laser initialization, where the laser diode and camera temperature controllers are activated. The laser diode current controllers can be activated 
while the camera is in focus allowing for simple activation of the laser diodes in the order necessary for a particular experiment. The simple user interface of the laser diodes allows for the user to simply turn on and off the lasers without having to adjust the current supplied to the diodes, which can lead to a change in output power of the laser diode. Thus by minimization of an error occurring due to adjustments made by the user to the current of the laser diode, the system is then more stable.

The second generation automated software (without co-registration) was then tested with a phantom using India Ink based absorption targets. The same 2\% Liposyn based phantoms with a $.45 \mathrm{cc}$ target were used. The target however was filled with India Ink in order to simulate the properties of a tumor more precisely. India ink is an absorbing agent; therefore the images collected will show the source distribution. However, upon subtraction with a background image (of a phantom without a target), the target will appear as the area with the smallest signal. This study determined the extent to which the second generation automated software was capable of functioning in comparison to the first generation imager in terms of target detectability, when using a uniform source strength system (as in second generation imager) versus a non-uniform source strength system (as in first generation imager).

\subsection{Co-registration}

The co-registration software that was used for the first generation probe head was not functional with the second generation system. Since the plate that attaches to the camera now has 192 fibers attached, where half of the plate is for the left probe head and the other half for the right probe head, the algorithm for converting the raw data to a $2 \mathrm{D}$ 
contour plot had to be modified as discussed in the previous section. Along with the instability and high cost of the ultrasound tracker, the two probe head design of the second generation imaging system would not be trackable by the ultrasound tracker without purchasing a second ultrasound tracker. Therefore the optical tracker was implemented to the co-registration software.

\begin{tabular}{|l|l|l|}
\hline \multicolumn{1}{|c|}{$\begin{array}{c}\text { Acoustic Co-registration } \\
\text { (First Generation) }\end{array}$} & \multicolumn{1}{c|}{$\begin{array}{c}\text { Optical Co-registration } \\
\text { (First Generation) }\end{array}$} & $\begin{array}{c}\text { Optical Co-registration (Second } \\
\text { Generation) }\end{array}$ \\
\hline Single probe and single phantom & Single probe head and single \\
phantom & $\begin{array}{l}\text { Two probe heads and ability to } \\
\text { have both probe heads on one } \\
\text { phantom or each probe head on a } \\
\text { separate phantom }\end{array}$ \\
\hline Configured for only reflectance & Configured for only & Probe head orientation can \\
since only one probe is used & reflectance since only one \\
probe is used & $\begin{array}{l}\text { correspond to reflectance and/or } \\
\text { transmittance }\end{array}$ \\
\hline Acoustic Tracking & Optical Tracking (2D) & Optical tracking (2D) \\
\hline Subtraction from background and & Subtraction from background \\
target image only & and target image only & $\begin{array}{l}\text { Subtractions can be performed } \\
\text { between target and background } \\
\text { images of the same probe head, or } \\
\text { subtract the probes from each other. }\end{array}$ \\
\hline
\end{tabular}

Table 3.2 Comparison of features of the first and second-generation co-registration software

Real time co-registration requires a tracker to be placed on each probe head to coregister the appropriate probe in its proper location. Tracking will be discussed in more detail in Section 3.10. The co-registration created for the second-generation probe includes the ability to use a different phantom for each probe head, thus separating the projections from the probe heads in each of the phantom meshes. The images are saved 
similarly to the original co-registration software as a png for later comparison or summations. Figure 3.7 above shows the co-registration flowchart and was described for the first generation optical imager. The difference towards the second generation imager was primarily the addition of a second tracking system and multiple meshes rather than a single mesh. The time to load the co-registration software is longer by an extra 2 minutes. However, loading the mesh is performed once prior to the beginning of imaging. The image collection and co-registration process take the same amount of time in first and second generation co-registration approaches.

Modifications in the software interface were made as follows:

- The MatLab code was modified to accept and display a single or two meshes depending on the experiment

- The tracking interface was changed from Ultrasound and MatLab based to Optical and LabView based as well as multiple tracker support with calibration

- The figures were modified to save each co-registered image individually for each probe head and mesh.

The second generation co-registration approach has the ability to individually show the probes on their corresponding phantom mesh and according to their respective view. Depending on which orientation or phantom study is desired (transillumination, reflectance on the same face or different faces, etc) the second generation co-registration approach has the option to give the initial positions of the probe heads on the mesh. Once the images are ready to be projected onto the mesh, each probe head has its own algorithm to determine the closest point from the probe patch to the phantom mesh 
allowing for individual projections and no possibility of co-registering the wrong image on the wrong mesh. The co-registered images are displayed on the 3D cubical phantoms. Here, two probe heads were simultaneously placed on adjacent sides of the same phantom (or alternately could be placed on two different phantoms). The displayed images are showing the same phantom in different viewing angles, such that both the coregistered images of the probe heads are visible to the viewer. The probe head image was separated into the different viewing angles not only to give the operator a clear view of the probe's image, but also because the co-registration process will only co-register a single probe's image onto the phantom if multiple images are present on the same phantom. The display would be similar when using two different phantoms and coregistering the probe heads, where always the viewing angle is adjusted such that the coregistered images are visible to the viewer. 


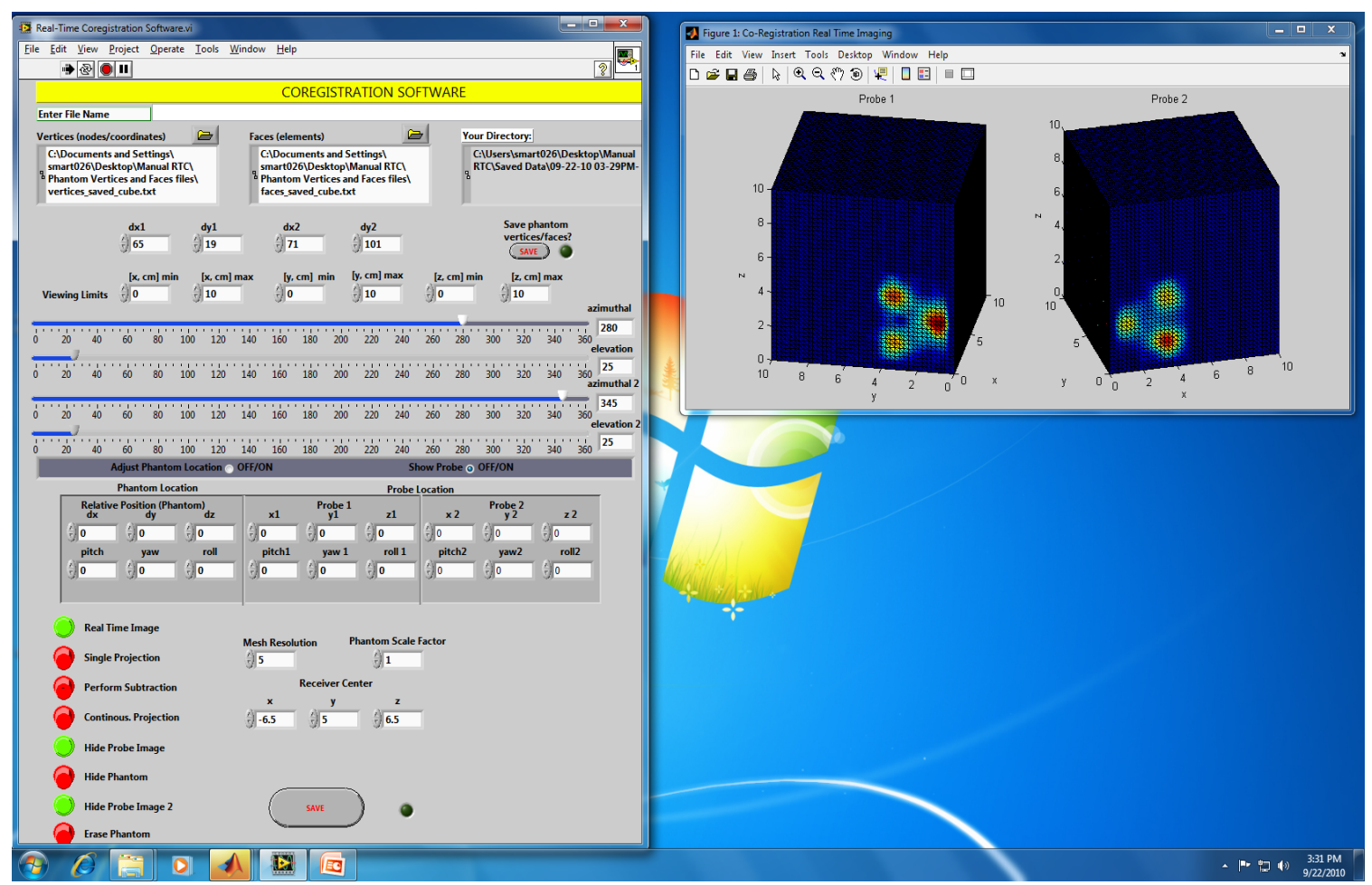

Figure 3.18 Second Generation Co-Registration Approach Software Front Panel

\subsection{D Co-registration Testing with Tracker}

Testing of the second generation co-registration approach with multiple trackers was performed similarly to the first generation tracker discussed in Section 3.4.4 for 2D tracking accuracy comparison with the first generation co-registration approach. Once the software was ready to be tested, the circuit developed for 2D tracking was duplicated and used on an two aluminum blocks to view the accuracy that the co-registration had of imaging with a dummy image for each probe view. A false image was loaded into the co-registration software, and a mesh of the cubical phantom was used. The aluminum blocks to be tracked were initialized at the bottom right of the cubical phantom (on one face) and on the bottom left of the phantom (on the other face). The false image was coregistered at the initial position to test the ability of projection and saving images. The 
aluminum blocks were then moved to simulate the use of the probe heads during a phantom study. The accuracy of the tracker was tested as well as the ability to project and save the image with the 2D tracking system integrated to the co-registration software. The results from the tracker were compared to the physically marked positions on the face of the cubical phantom.

\subsection{Tracking}

The second generation co-registration approach with the $2 \mathrm{D}$ trackers was then adapted for the two probe heads. Both probe heads are tracked individually and in real time allowing for a simultaneous method of co-registration and imaging for both probe heads. A single LED circuit and bracket was created to hold the LED's on each probe head. Since the 2D tracking requires the LED to be a known distance away from the tracking detector, a bracket was also made for the Wiimote to hold it in place and reduce any possibility of movement for the tracker, which would introduce error. The same algorithm for 2D tracking in the first generation system was duplicated for each probe head and implemented into the second-generation co-registration approach. An existing sample image was loaded into the co-registration software (without acquiring any new images), and a mesh of the cubical phantom was used. The probe heads were initialized at the bottom right of their respective cubical phantoms and a previously recorded image was co-registered in position. The probe heads were then moved in a linear fashion as in a phantom study. The Wiimote was placed a distance of $32 \mathrm{~cm}$ from the LEDs and the probe heads were moved to 10 different $(\mathrm{x}, \mathrm{y})$ positions for each distance on a $10 \mathrm{~cm} \times$ $10 \mathrm{~cm} \times 10 \mathrm{~cm}$ cubical phantom for each probe head as shown on table 3.3 . The accuracy 
of the tracker was determined, while testing the effectiveness of the co-registration software as well. In order to differentiate between the two probe heads' trackers, an identifying variable known as a 'handle' is assigned to each Wiimote (i.e. the tracker) by Labview. By assigning a handle to each probe's tracker, the identity of each probe is preserved in the software. The accuracy of the tracker through the co-registration software was found through this experiment and compared to the single object optical tracking and ultrasound tracking. The time of imaging and co-registration was not changed in multiple object tracking since the objects were tracked and co-registered simultaneously.

\begin{tabular}{|l|l|l|}
\hline X Location $(\mathrm{cm})$ & Y Location $(\mathrm{cm})$ & Repetitions $(\mathrm{cm})$ \\
\hline 0 & 0 & 5 \\
\hline 0 & .5 & 5 \\
\hline 0 & 1 & 5 \\
\hline 0 & 1.5 & 5 \\
\hline 0 & 2 & 5 \\
\hline 1 & 2 & 5 \\
\hline 1 & 1.5 & 5 \\
\hline 1 & 1 & 5 \\
\hline 1 & .5 & 5 \\
\hline 1 & 0 & 5 \\
\hline
\end{tabular}

Table 3.3 Positions collected in the 2D tracking study for the second generation probe heads. Both probe heads were moved in the same positions on separate phantoms where $(0,0)$ is the bottom right position of the phantom. Therefore each of these positions was repeated 5 times per probe head. 


\subsection{D Co-registered Imaging Testing}

The second-generation optical imager was used in phantom studies for verification and comparison to the first generation optical imager. The tracker's accuracy during use in imaging was compared to the physical position, as well as the ultrasound tracker, and optical tracker alone separate from the probe heads from the previous studies. The data was collected and evaluated and compared to the first generation images. All the images were collected from a $10 \mathrm{~cm} \times 10 \mathrm{~cm} \times 10 \mathrm{~cm}$ phantom $2 \%$ Liposyn phantom. The target used was a $.08 \%$ (by volume) India Ink .45 cc target placed at $1 \mathrm{~cm}$ depth in the center of the phantom. First, one probe was placed on a phantom with a target and the second probe head on a phantom with no target. The images were collected at $0,1,2$, and $3 \mathrm{~cm}$ in height with respect to the origin (which is the bottom right corner of the phantom on the face being imaged). The images were co-registered and the positions recorded for comparison. Then the collection of the data was repeated with the target in the opposite phantom. The last study involved both probe heads, next to each other on the same face of the phantom with a target and collecting images at heights of 1,2 and $3 \mathrm{~cm}$. The study was performed to determine if the saved coregistered images accurately depicted and located the two probe heads on the phantom face. In addition the imaging and co-registration time of the second generation imager was compared to that of the first generation imager in this study.

\subsection{Summary}

The first generation optical imager required many upgrades to the software in order to optimize its efficiency. Firstly, the combination of all the different software 
packages had to be performed in order to obtain the fastest method of imaging with the least amount of errors caused by computational or human error. Secondly, the tracking had to be improved in order to obtain a much more accurate and stable method of locating the probe on a phantom. Three different trackers were tested, but only two were determined to meet the criterion for effective tracking. Optical tracking however, has been far more stable than ultrasound (or acoustic) tracking, and thus carried over to the development of the second generation optical imager.

The development of the second generation optical imager was a multi-student project involving both hardware and software development. Many of the first generation imager's attributes were carried over, however they were improved upon. The software involved a new automation technique for a new camera, multiple laser sources, and multiple probe heads. The co-registration approach also took on a new technique of projecting and saving the images with the new arrangement. Finally, tracking was integrated to the second generation probe and was tested on phantom studies in order to determine if it were functional. The results will be discussed in Chapter 4 . 


\section{Chapter 4: Results and Discussion}

The assessment of automation and tracking devices will be discussed in detail in this chapter. The results obtained from the methods described in Chapter 3 will determine the improvements through automation as well as a potential alternative to the acoustic tracker. Automation of the software used to acquire images and co-register the images will ideally lead to less time for completion of image collection and coregistration. Through the use of a more stable/accurate tracking devices, the accuracy of 3D reconstructions may be improved and can be tested in later experiments.

\section{Part I Modifications Towards the First Generation Optical Imager}

\subsection{Study 1: Automated Software}

Automation of the co-registration was tested in order to assess the improvements in time of acquisition and co-registration. The time for the original multi-software package described in Section 2.5 to store a co-registered image was more than 5 minutes. After automation, the time for each image to be acquired, co-registered and stored was 35 seconds on an average. Therefore the time improvement through automation led to a $90 \%$ decrease for imaging. The graphical user interface provided a simpler method of use over the previous software since all the controls can be found in the same window. The automation provided for minimal user input, reducing the amount of human error that was possible through the use of multiple software (as in the previous version). Although the co-registration was improved, the error of tracking was still prevalent. Therefore the assessment of the trackers (described in Section 3.3) was performed. 


\subsection{Study 2: Tracker Evaluation Results}

The electromagnetic, acoustic, and optical trackers were evaluated for accuracy and efficiency in data collection for the location of a point in space. The results discussed below explain the determination of which tracker would be the best solution for our imager.

\subsubsection{Electromagnetic vs. Acoustic Tracking Results}

Figure 4.1 illustrates the results of the studies described in Section 3.3.2 and 3.3.3, comparing the performance of the electromagnetic and acoustic tracker data acquired on the face of the phantom. The electromagnetic tracker showed a large fluctuation in recorded positions when metallic objects were present near the system. To reduce the amount of error from walls and other metallic objects, the metallic objects should be spaced approximately 3 times the distance between the receiver and transmitter. Our current imaging system involved multiple metallic components in the vicinity of the receiver and/or transmitter. Hence, the implementation of the electromagnetic tracker will further reduce the accuracy of co-registered imaging. On the contrary, the currently implemented acoustic tracker (to our hand-held imager) has significantly higher accuracy than the electromagnetic tracker, as observed from the range of errors in tracked location with respect to the true location (see Table 4.1).

The errors described in Table 4.1 were determined through two methods. The first method involved measuring the distance between the true position of the receiver and the position given by the tracker given by equation 4 :

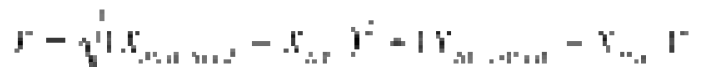

Equation 4 
where " $r$ " is the distance between the true position of the receiver and the given position of the receiver given by the tracker. Also, " $\mathrm{x}_{\text {true }}$ " and " $\mathrm{y}_{\text {true }}$ " are the true $(\mathrm{x}, \mathrm{y})$ positions of the receiver, " $\mathrm{x}_{\text {measured" }}$ " and " $\mathrm{y}_{\text {measured" }}$ " are the given $(\mathrm{x}, \mathrm{y})$ positions of the receiver given by the tracking device. Then the average of these distances was given in the table along with the standard deviations. The second method involves using an arbitrary reference point, $(-1,-1) \mathrm{cm}$, where the distance of each of the true positions of the receiver and the measured positions of the receiver given by tracking device can be measured. This approach was employed in order to compare the accuracy of the coregistered points with respect to a reference point, in terms of the error in true and measured distances w.r.t. the reference point. These distances are given by equations 5 and 6:

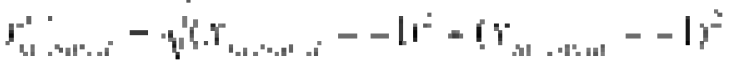

$$
\begin{aligned}
& \text { Equation } 5
\end{aligned}
$$

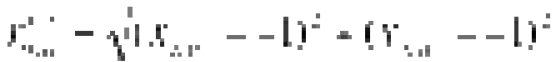

$$
\begin{aligned}
& \text { Equation } 6
\end{aligned}
$$

where " $r$ ref measured" is the distance between the positions of the receiver and the reference point, $(-1,-1) \mathrm{cm}$ given by the tracking device and " $\mathrm{r}$ ref ${ }_{\text {measured" }}$ " is the distance between the true positions of the receiver and the reference point, $(-1,-1) \mathrm{cm}$. Also, " $\mathrm{x}_{\text {true }}$ " and " $\mathrm{y}_{\text {true" }}$ " are the true $(\mathrm{x}, \mathrm{y})$ positions of the receiver, " $\mathrm{x}_{\text {measured" }}$ " and " $\mathrm{y}_{\text {measured" }}$ are the given $(\mathrm{x}, \mathrm{y})$ positions of the receiver given by the tracking device. Once the reference distances were known, the relative error can be determined for the tracking devices (see equation 7). Table 4.1 tabulates the average relative errors for the tracking devices along with the standard deviation of the errors:

$$
r^{2}
$$


Even in the absence of metallic objects around the electromagnetic tracker, the error in the tracked location was as high as 50\%, making electromagnetic tracker inappropriate for our application. Although the points collected for the ultrasound tracker seem very accurate, it was observed that when the receiver was held at a particular point in space untouched, there was an oscillation around the true position of the receiver with a difference of approximately $\pm 0.5 \mathrm{~cm}$. Although it may not be a large fluctuation, it can introduce error into the co-registration of each image onto the mesh, and eventually in the detected target's (or tumor's) location.

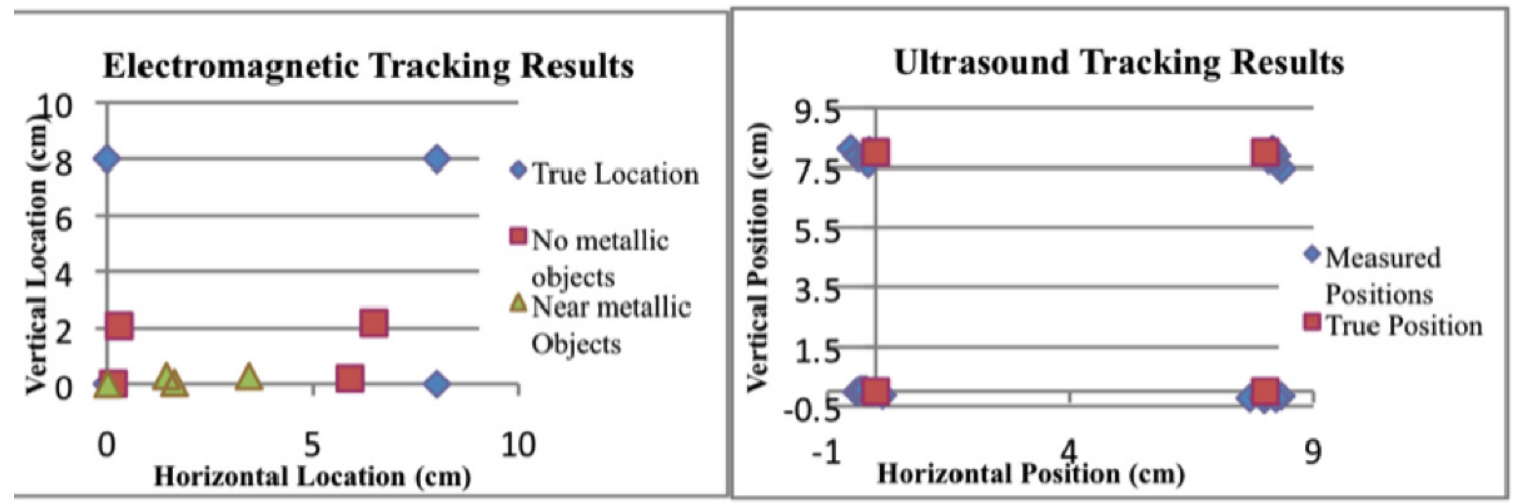

Figure 4.1 Comparison of the results obtained from the electromagnetic tracker and the acoustic tracker.

The transmitter end of each tracker was moved along the four corners of the face of a cubical phantom.

\subsubsection{Optical Tracker Limits}

The limits of the camera's visibility for the IR LED allow for the user to determine the optimal field of operation for any given experiment. Since each experiment, whether it be phantom studies or human subject studies, require different field of operation due to the size of that object which is being scanned. In Section 3.4.3, the field of view of the Wiimote was determined to be $43^{\circ}$, while the viewing limit was a distance of $173 \mathrm{~cm}$ from the Wiimote. Therefore, in order to perform a successfully 
tracked experiment, the operation of the LED must be contained within the limits of the Wiimote. Ideal scenarios for phantom studies and human subject studies would be to maintain the whole of the area of interest within the field of view of the Wiimote. Due to the fact that multiple IR LEDs may be used to achieve greater degrees of freedom (DOF), the distance at which the LEDs are placed from one another depend on how far the Wiimote is from the LEDs. For example, a single LED gives an $(\mathrm{x}, \mathrm{y})$ position, however it gives no description of the angle of rotation. A second LED added to an object would allow for the rotation about the $\mathrm{z}$ axis (depth) to be known. A third LED would ideally give information on as much as 6 DOF since it can give information on the depth and rotation about each the $\mathrm{x}, \mathrm{y}$ and $\mathrm{z}$ axis or pitch, yaw and roll (this was the basis of the attempted 3D tracking described in Appendix 1). In the current work, only 2D optical tracking approach has been successfully demonstrated, with the 3D optical tracking as an immediate future direction.

\subsubsection{Optical vs. Acoustic Tracking Results}

Figure 4.2 shows the results of Section 3.3.3 and 3.3.4, comparing the performance of the optical and acoustic tracker data acquired on the face of the phantom. Both trackers were capable of accurately tracking the true location as seen from Figure 4.2 and Table 4.1. When observing the measurements obtained from the acoustic and optical trackers, the acoustic tracker has variations/fluctuations during movement and also at a particular location. However, the optical tracker had no fluctuations when positioned and maintained at a particular location, demonstrating its stability over the 
acoustic tracker. It has been demonstrated that the optical tracker is accurate and stable in 2D tracking in comparison to the acoustic tracking device.

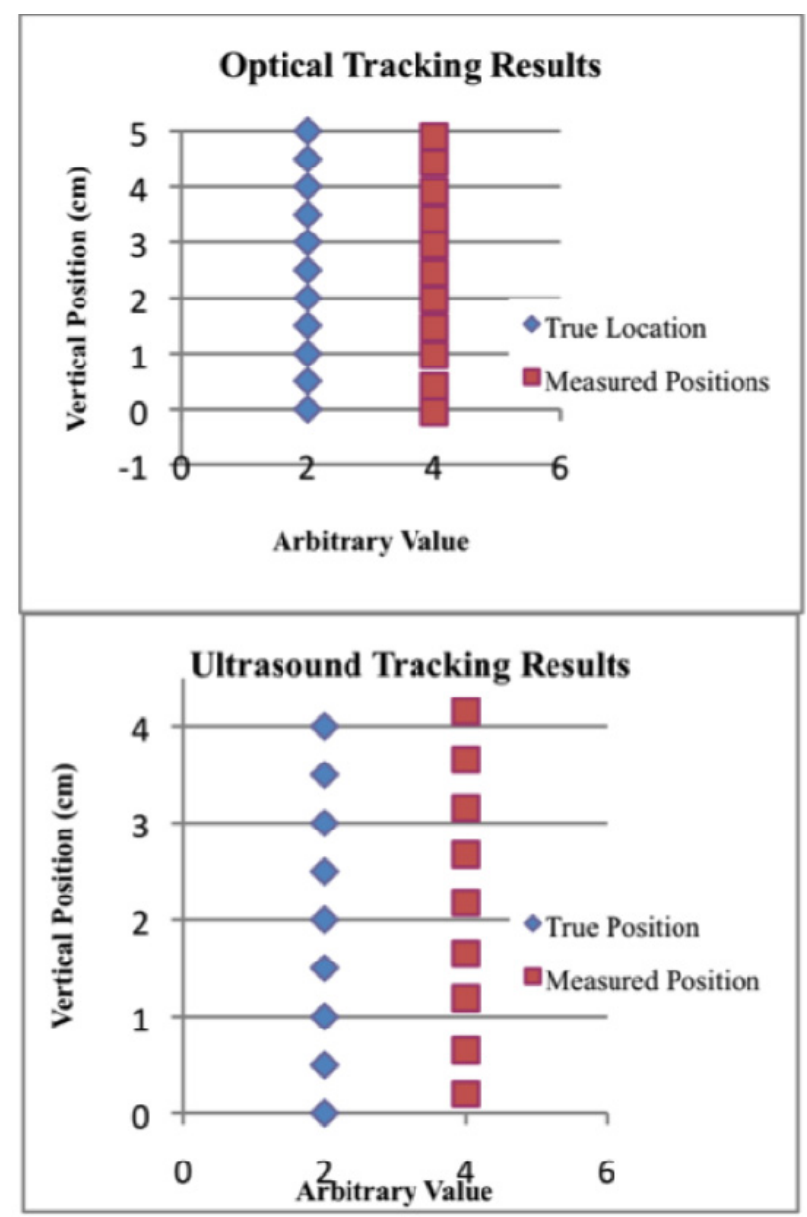

Figure 4.2 Comparison of tracked positional information using an optical and acoustic tracker. The LED (optical tracker) and transmitter (ultrasound tracker) were moved in a linear fashion along the face of a cubical phantom. Positions were recorded at $.5 \mathrm{~cm}$ increments. 


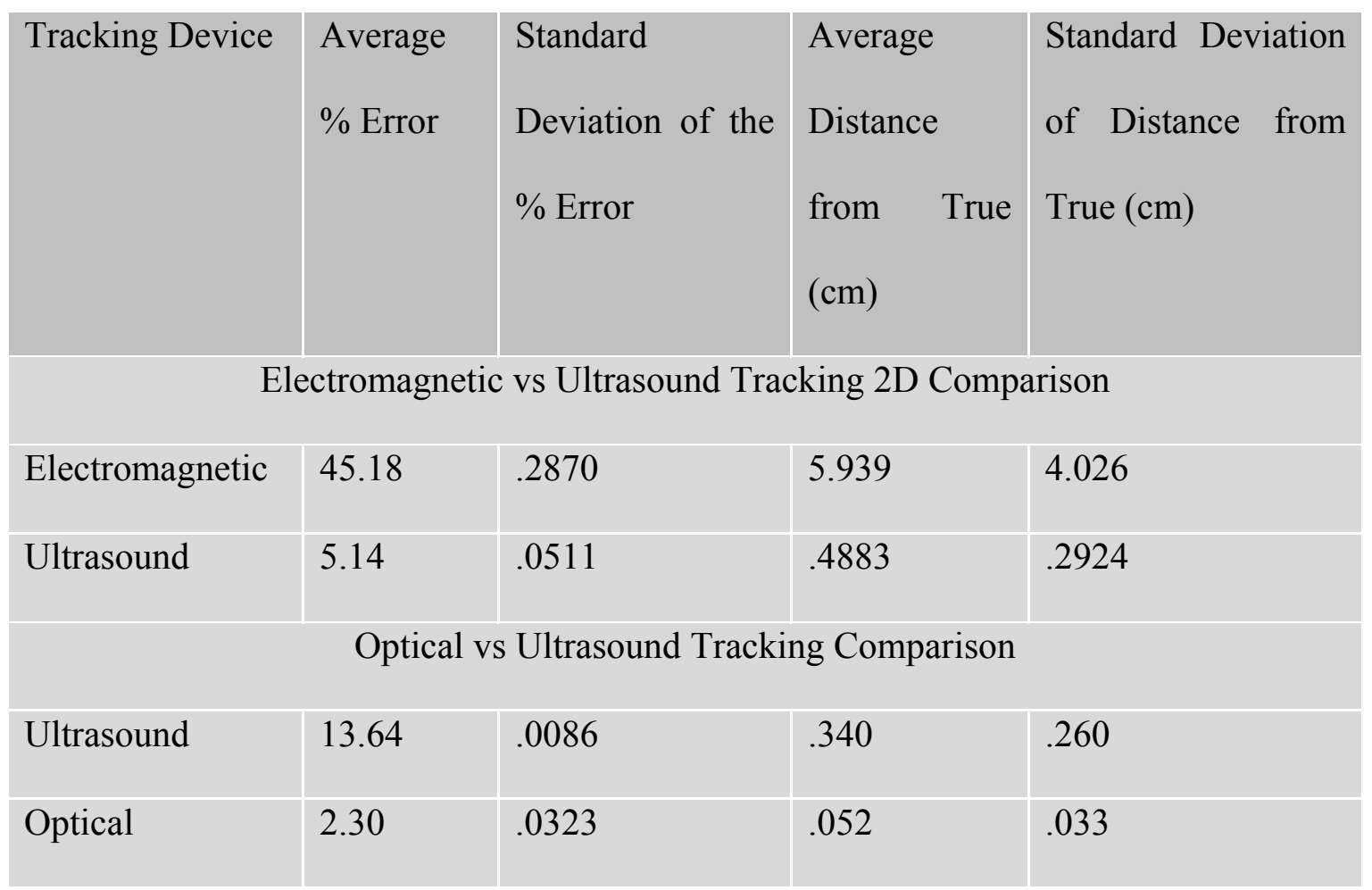

Table 4.1 Error comparison of the tracking devices in two dimensional tracking. The averages of the errors are shown here as well as the average distance from the true measured position as calculated using Equations 3 - 6.

\subsection{Optical and Acoustic Tracker 2D Co-registration}

Testing of the acoustic tracker and optical tracker was performed individually and the results show that the acoustic tracker would be less accurate and stable than the optical tracker. In order to check if there was some effect from the error on the position of a target, images were collected and co-registered using both acoustic and optical techniques with the first generation optical imager. The position of the probe head on the mesh was compared, as was the position of the target within the phantom given by the images through the two tracking techniques. The target was placed at $9 \mathrm{~cm}$ in the horizontal direction and $5.5 \mathrm{~cm}$ in the vertical direction. Figure 4.3 below shows the coregistered images collected where each box is at a different position. Through these 
images it may be observed that the target is not detectable as the probe head is moved away from the target location. As the probe is moved closer to the bottom of the phantom, it can be noted that the source distribution becomes much more apparent than does the signal from the target, since the target is out of the line of sight of the probe head. When a fluorescent target is present in a phantom, the signal obtained from the target is much larger than the signal from the sources. Once the target is no longer in the line of site of the probe head, the source signal becomes visible and dominant in the collected images since there is no signal coming from the target in that area. 

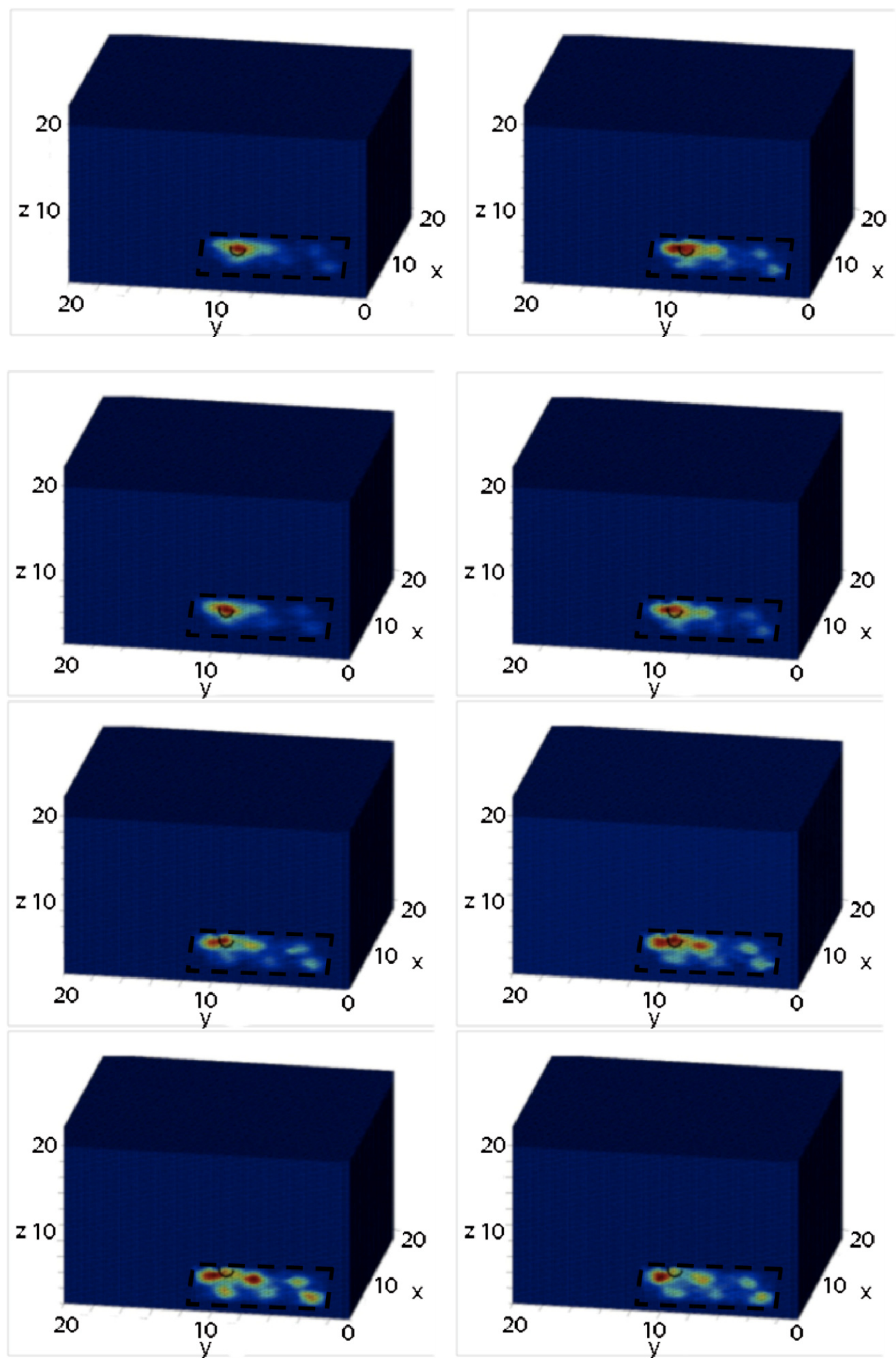

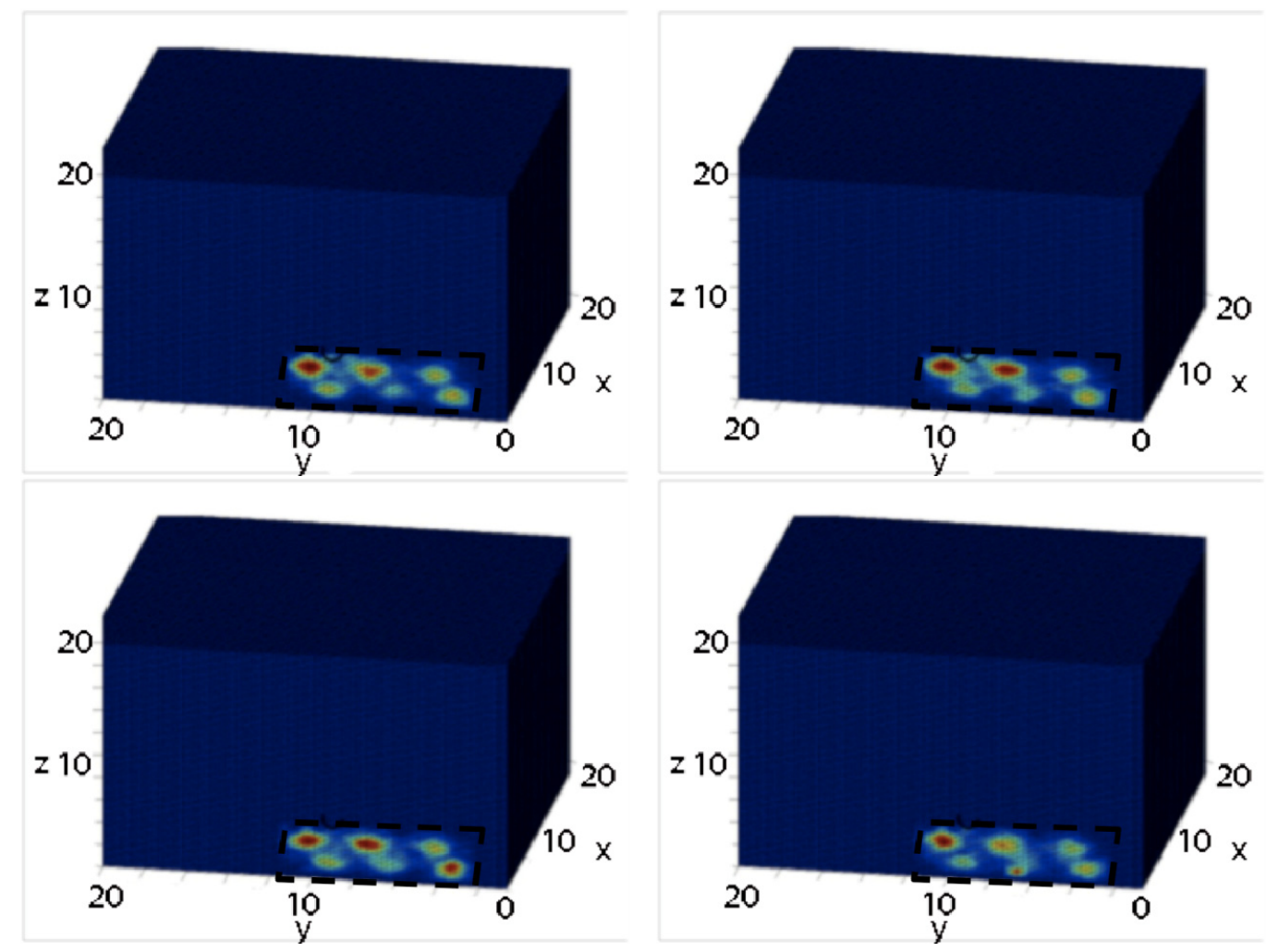

Figure 4.3 Co-registered imaging demonstrating the detection of a target as the probe head is moved in different positions along the face of a cubical phantom. The black circle represents the position of the.45 cc ICG target within a 2\% Liposyn filled cubical phantom. The dotted black box is the outline of the probe head.

The table below shows the average errors and standard deviation where the average errors for target location for both tracking devices was shown to be significantly different for the two trackers, which will have an effect on correctly identifying the location of a tumor within the breast tissue in future in vivo studies. 


\begin{tabular}{|l|l|l|l|l|l|}
\hline Tracking Device & Average & Standard & Average & \multicolumn{2}{l|}{ Standard Deviation } \\
\hline & \% Error & Deviation of the & Distance & of Distance from \\
& & from Error & True & True $(\mathrm{cm})$ \\
\hline Ultrasound & 3.1 & .0107 & .347 & .110 \\
\hline Optical & 0.8 & .0066 & .127 & .093 \\
\hline
\end{tabular}

Table 4.2 Comparison of the target location and errors of the probe location for both trackers. The location of the target was taken from the images that were collected in the $2 D$ studies with a $2 \%$ Liposyn filled cubical phantom. The true location of the target was compared to the location found in the images giving the errors shown above.

Although the standard deviations for the collected data were low, the stability of the acoustic tracker was noticeable. The standard deviation continues to increase when a larger data set is collected due to the instability of the position of the probe head given by the acoustic tracker. Therefore, although the optical and acoustic both have similar errors on the target position within an image, the acoustic trackers instability causes it to be unreliable for multiple use and larger data sets.

\subsection{Optical Tracking LED Affect on Imaging}

In the study discussed in section 3.6, the optical tracker was analyzed for the amount of noise, if any, the infrared LEDs would have on acquired images. The images obtained in this study did not show any signal due to the LEDs as seen in the figure below. Images obtained with the LED off had a negligible difference to the images obtained with the LED on, as seen in the subtracted image where the maximum value is 600 arbitrary units (this is below the noise level of 4000 arbitrary units). Therefore, it has 
been determined that the optical tracker's infrared LED's will not have an effect on the collected images.
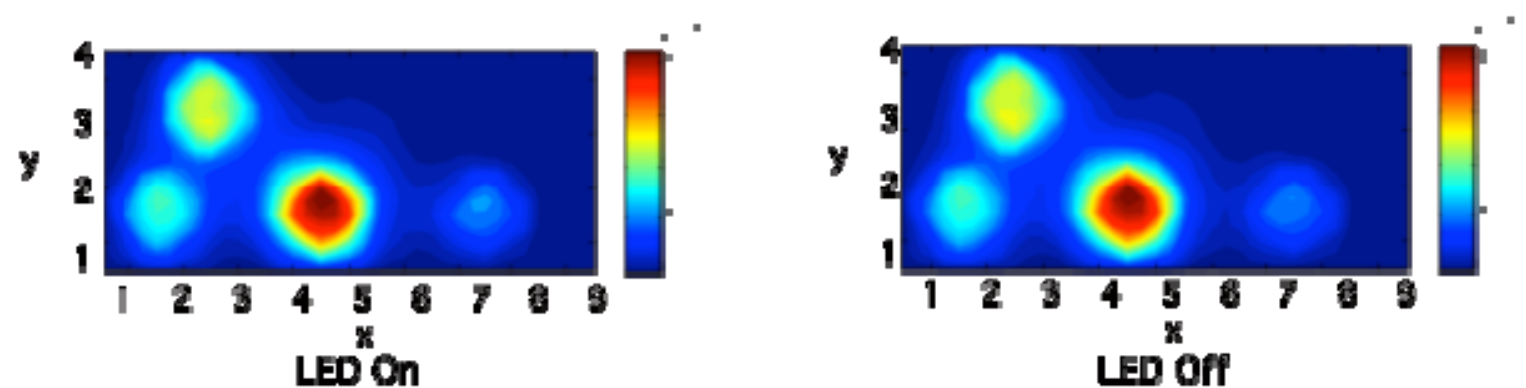
LED OfP

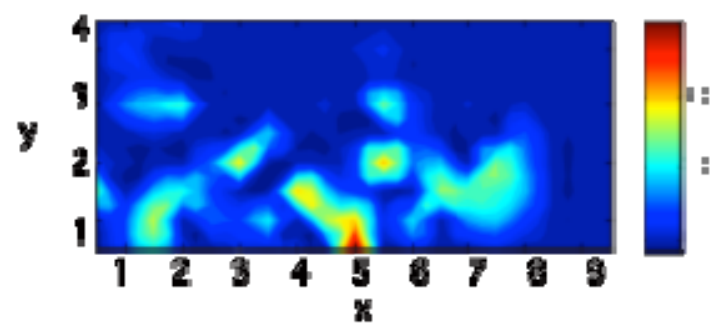

Subtracted (LED On - LED Off)

Figure 4.4 2D Contour Images of intensity signals acquired from the phantom surface under the following conditions: (A) When the LED located on the hand-held probe were turned off, (b) Comparing the effect of the Tracker's LED On and Off. The LED off image involved the LED being attached to the probe but not powered while the LED on image involved the LED being turned on while attached to the probe. The subtracted image involves the subtraction of the LED on image from the LED off image giving the difference of the two images.

\section{Part II Modifications Towards Second Generation Optical Imager}

\subsection{Second generation Optical Imager}

The construction of the device was completed amongst all the members of the lab. The source distribution is evenly distributed to all six source fibers at $2 \mathrm{~mW}$ each. The distribution allows for a much more effective method of target detection in phantom 
experiments. The automated software was developed for the second generation optical imager where different probe head design had to be taken into account. The time of imaging with the second generation probe was comparable to the first generation at approximately 35 seconds to collect and co-register an image. A similar user interface was used for the second generation imager to keep the simplicity and user-friendly structure available from the first generation imager.

Original testing of the imaging software developed for the second generation optical imaging system was performed without co-registration so as to test the ability to collect images and detect targets as described in Section 3.11. The following image shows the target and background of a particular phantom study. Only the source distribution can be seen, and no difference is immediately visible. However, upon subtraction, the India absorbing Ink target, is clearly visible in it's appropriate location. This study displays the ability of the second generation system to function accurately in the detection of an absorbing agent. Although the target is not detected in the target images when originally taken, the target is seen once the subtraction of the background is performed. In Figure 4.5, the background image (the image where there is no target located in the phantom) was subtracted from the target image (the image where the target is located in the phantom), and the area in which the target is located (with least intensity) becomes visible. However, if the subtraction were performed in the reverse order, the target is given by the area with the most intensity. The color scheme shows either the area where there is the most light intensity present (represented by red) or the area where there is the least intensity (represented by blue) and scaled for all other intermediates in intensity. When an ICG target is used, the target location is represented by the high 
intensity emitted signal (shown as red regions in Figure 4.3) since more intensity is coming from the fluorescent target than the background. However the India Ink target (which is an absorbing agent) would be a low intensity or a highly absorbed signal (shown as blue regions in Figure 4.5). In other words, a phantom containing a fluorescence emitting target (using a fluorescence based contrast agent) would show a higher intensity signal emitted from the target, upon subtracting the background signal. On the contrary, a phantom containing a highly absorbing target (using absorption based contrast agent) would show an increased absorption or a relatively low intensity signal from the target region, eve upon subtracting the background signal. This study simulates future bilateral studies that are to be performed on a human subject study where the lesion locations are unknown. However when images from left and right breasts are subtracted, ideally the researcher can determine on which breast a lesion can be found. Thus the experiment provided sufficient information to show the functionality and effectiveness of the second generation optical imager along with the newly modified automation software for use in phantom and in vivo studies. 
Figure 4.52D surface contour plots of intensity distribution acquired on two $10 \times 10 \times 10$ cc cubical phantoms using the 2 probe heads of the Gen-2 imager. A $0.45 \mathrm{cc}$ absorption contrasted target filled with $.08 \%$ by volume India Ink was placed in Image collected with the second generation imaging system. The top image displays the images taken with the target in place. The center images display the images taken of the phantom without the target in place. Finally the subtracted images show the lesion location once the background is subtracted from the target. 


\subsection{Co-registration with the Second Generation Optical Imager}

Once the second generation optical imager was functional, the co-registration was modified to be implemented to the second generation automated software as described in section 3.11. The figures below show samples of images collected and co-registered using the software and second generation imager in phantom studies.
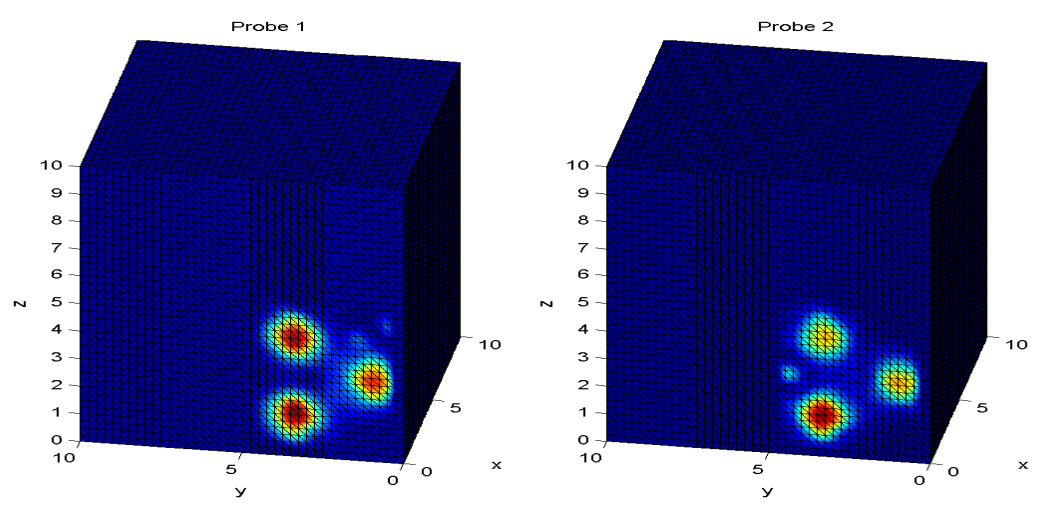

Figure 4.6 Co-registered images from the second generation optical imager. The image on the left corresponds to probe 1, and the right to probe 2. Each probe was on a separate cubical phantom and both images were collected with the same camera at the same time. The image is a previously recorded image and does not represent any signal coming from the probe heads or the phantom.

In figure 4.6, the red regions represent the source distribution of the second generation probe since no target was in the phantom, where the images collected would show the source distribution. When compared to the first generation's actual source distribution, the second generation is much more evenly distributed and consistent. Also it may be noted that the second generation probe head is smaller than the first generation optical imager so it covers a smaller area of the phantom than does the first generation imager. The co-registration software is capable of imaging with both probe heads 
simultaneously while processing the optical data with a single camera and separating the collected image of optical data onto separate phantoms corresponding to the appropriate probe head. Each probe can be imaged on the same phantom or on separate phantoms, allowing for the simplest way of performing any combination of experiments.

\subsection{D Tracking with the Second Generation Optical Imager}

The tracked positions of the probe on the phantom mesh had an error in accuracy of distance from the true location of $.126 \mathrm{~cm}$ and a standard deviation of .103 . This is comparable to the accuracy of the first generation as shown in Table 4.3 and is consistent with the stability that was seen in earlier experiments. This accuracy is much more stable than what is seen in the acoustic tracker for the first generation probe head. This technology of optical tracking is very promising towards improving the accuracy of the system towards $3 \mathrm{D}$ tracking in the future.

\begin{tabular}{|l|l|l|l|l|}
\hline $\begin{array}{c}\text { Co-Registration } \\
\text { Technique }\end{array}$ & $\begin{array}{c}\text { Average } \\
\% \text { Error }\end{array}$ & $\begin{array}{c}\text { Standard } \\
\text { Deviation of } \\
\text { the \% Error }\end{array}$ & $\begin{array}{c}\text { Average } \\
\text { Distance from }\end{array}$ & $\begin{array}{c}\text { Standard Deviation } \\
\text { of Distance from }\end{array}$ \\
\hline Ultrasound & 5.24 & .0331 & .524 & \multicolumn{1}{c}{ True (cm) } \\
\hline Optical ( $1^{\text {st }}$ Gen.) & 1.76 & .0174 & .227 & .104 \\
\hline Optical $\left(2^{\text {nd }}\right.$ Gen.) & 3.34 & .0305 & .126 & .103
\end{tabular}

Table 4.3 Table showing the error of the acoustic, optical, and multiple objects with optical. Each of the co-registration software's accuracy was compared in a study where the probe head with the tracker attached was moved along the face of a cubical phantom. The positions at which the probe(s) head was/were located were measured physically and through the co-registration software to provide an accurate comparison of the accuracy of each tracker. 


\subsection{Summary}

The automation of the software led to a significant decrease in time for coregistered image storage. The improvement of time for image collection and coregistration has not only reduced time, but also made the imager software more user friendly. Through the minimization of user inputs via automation, a possible reduction in the occurrence of user related errors simplifies the use of the optical imager.

With regards to the tracking devices, the electromagnetic, acoustic and optical tracking devices were all assessed towards the improvement of tracking stability. Electromagnetic was determined to have a large amount of error due to metallic objects near the device, while acoustic and optical had accurate results. Although both acoustic and optical trackers had similar accuracies in tracking, it was noted that the optical tracker was much more stable and a better alternative to the acoustic tracker for accurate 2D imaging studies.

The second generation optical imaging system has shown that it can be used to detect a target in a phantom effectively, through subtraction, and 97\% accuracy in target location detection. The optical tracker used towards the second generation approach has a low deviation from the actual position by an average difference of $.227 \mathrm{~cm}$ in comparison to acoustic tracked first generation approach of $.524 \mathrm{~cm}$ error. The decreased error is due to the stability of the optical tracker. However when multiple objects were tracked with the optical tracker, it was noted that the difference was further reduced to $.126 \mathrm{~cm}$. In addition to improving the accuracy and stability of the co-registration approach, the improvements made to the second generation imaging system has improved its detection capabilities and effectiveness. 


\section{Chapter 5: Conclusions and Future Work}

\subsection{Conclusion}

NIR imaging is a useful technique for deep tissue imaging and provides an alternative to the current cancer diagnostic techniques used in the clinic today. Optical imagers have classically been bench top imagers and only in the recent decades have there been advancements towards hand held optical imagers. Although current hand held imagers have been used in extensive research, none have been capable of 3D tomography. FIU's Optical Imaging Lab (OIL) has developed a hand held optical imager that has proven to be effective at 3D tomographic imaging using a hand held probe using co-registration techniques and an acoustic tracker. However, OIL's hand held imager had the following drawbacks:

1. Lack of full contact with tissue

2. Uneven source distribution

3. Multiple software co-registration

4. Unstable positional tracking.

The objective of this research is to improve the imaging time by automating the software approach into a single software package and improving the accuracy by either using a different tracking technique or improving the current tracking system. A LabView based graphical user interface was developed to address the time of imaging and multisoftware approach. By using LabView a single software would automate the entire imaging process from the camera initialization and image acquisition to coregistration onto a 3D mesh of the object or subject imaged and saving the images. The 
newly optimized automated software for the optical imaging system has proven to be effective in phantom studies. The time of imaging has been improved by $90 \%$ allowing for near real time imaging and co-registration, thus reducing the total time of imaging for a single subject, or phantom to approximately 3 hours, from multiple days.

The stability of the tracking system was addressed by testing acoustic, optical and electromagnetic tracking approaches. After much testing, the optical approach had proven to be the most stable and most accurate. The optical tracker was customized to track in $2 \mathrm{D}$ for the first generation optical imager. A conversion equation was determined to change the pixel values given by the optical tracker for an LED into the distance values to allow for $2 \mathrm{D}$ tracking. The error due to the instability of the tracking approach improved from $13 \%$ to a $2 \%$ average error. Although it may seem a small change, the error has improved the co-registration accuracy by means of a more stable tracking device.

In addition to developing the automated software and improving the tracking approach, the development of a second generation optical imager was addressed by colleagues. Their work included the integration of a multichannel source controller and the development of a smooth two part probe head. As part of my thesis, the automated software and tracker were modified to function with the new two probe head design. The tracker was added to the second generation probe head for $2 \mathrm{D}$ imaging in phantom studies and the modification of the co-registration was performed. A dual probe view design was used in the co-registration to allow the user to view the image on the phantom corresponding to the view of the probe head of interest as well as to separate the probes into two phantoms if a bilateral study is performed. In addition to a dual probe head view 
for independent views of each probe head, the co-registration software required that the two probe heads be separate in order to project the image to the phantom rather than having both images on the phantom simultaneously. With both images simultaneously being co-registered to the same phantom, the co-registration software was incapable of co-registering both images. Eventually, the dual probe head view allowed for the images to be co-registered simultaneously (but viewed on duplicate phantoms for each tracked probe head), and thus improve the overall imaging time and simplify the co-registration process. Co-registration has been improved to attain a better accuracy and faster imaging time necessary for successful detection of a target within a tissue phantom and can similarly be used in a human subject study.

Although 3D optical tracking was not completed in this work, acoustic tracking has been used to track the first generation optical imager in human subject studies. Multiple in vivo subjects have been imaged over the past few weeks using the automated software that was developed for the first generation optical imaging system. The coregistration and automated software has allowed for fast collection of the images and simple processing for subtraction and preliminary results from the images collected can be seen immediately. The imaging process for an in vivo subject with the software prior to automation was a lengthy process, upto 3 days for co-registered images. However, after automation, the time for a full imaging session of 8 images has been improved to 20 minutes with co-registered images.

The second generation optical imager is currently being used with co-registration on phantom studies in 2D. However, it has shown to be effective in detecting targets and has two probes for simple bilateral studies. The second generation optical imager has 
also just begun to perform clinical studies on subjects, but results have not yet been processed. The second generation optical imager allows the operator to place one probe head on each breast simultaneously and acquire the images at the same time. Ideally this would allow for half the imaging time of the first generation imager, but that has yet to be tested. The second generation optical imager has the capability of performing all the first generation processing techniques in half the time. The capabilities of the second generation optical imager along with the modified automated software and the modified optical co-registration software will be a useful clinical tool. It is capable of fast, noninvasive, and comfortable breast cancer imaging, which has potential for diagnostic/prognostic applications of the disease with maximal patient comfort and low imaging costs.

\subsection{Future Work}

The work performed towards the completion of my thesis has led to improvements in the first generation optical imaging system through the automation of image acquisition and co-registering the collected image. Improvements made to increase the accuracy of the co-registration software have proven to be effective in phantom studies with the first generation optical imager and recently with the second generation optical imager. Clinical in vivo studies have been performed with both first

and second generation imaging systems. However the second generation optical imager has not yet been tested with the automated co-registration software for in vivo studies. Through clinical in vivo studies, comparisons between the first generation optical imager 
and the second generation optical imager's effectiveness in diagnosing breast cancer can be made.

In Appendix 1, the development of the 3D tracking technique using the optical tracker is described. Although the research and development carried out towards completion of the 3D tracker was incomplete, the research already carried out should lead future work in the proper approach to solving the 3D tracking problems. Once the optical tracker has been completely customized and its stability and accuracy demonstrated for 3D positional tracking, it will be implemented on the automated co-registration software. The co-registration software has already been modified to accept any tracker, which will simplify the integration of the 3D optical tracker.

During in vivo studies, it may also be necessary to track the tissue geometry being imaged, since the subject can potentially move during the studies. Recently the method of subject positioning is more in an inclined position, which reduces movement from the subject. However, there is still the possibility of the subjects' chest cavity movement due to breathing. The movement is very minimal and will have little to no effect on coregistration, since the co-registration process projects images taken in the position closest to the tracked position on the mesh. This alone does not justify not tracking the subject; but it does present the necessity of performing extensive studies to determine the effect of breathing on the position of the co-registered image and any effect breathing may have on tumor locations.

The implications of the work performed in this area have been to completely translate the hand held imaging technology to the clinic. The work performed up to this time has been very promising and continuing studies are only supporting the 
effectiveness of the hand held optical imager. Such technology will provide an additional early stage diagnostic tool, which potentially can reduce the amount of unnecessary exposure to harmful radiation and number of biopsies. The hand held optical imager is a very promising tool towards breast cancer diagnostics and may be widely accepted in not only the field of breast cancer diagnosis but in other fields such as brain mapping and other non-invasive diagnostic fields. 


\section{REFERENCES}

1. A. Cerussi, N. Shah, D. Hsiang, A. Durkin, J. Butler, B.J. Tromberg. "In vivo absorption, scattering, and physiologic properties of 58 malignant breast tumors determined by broadband diffuse optical spectroscopy." J Biomed Opt 2006;11(4):044005.

2. A. Cerussi, N. Shah, D. Hsiang, A. Durkin, J. Butler, B.J. Tromberg. "Predicting response to breast cancer neoadjuvant chemotherapy using diffuse optical spectroscopy." Proc Natl Acad Sci USA 2007;104(10):4014-9.

3. American Cancer Society. "Cancer Facts and Figures 2010." Atlanta: American Cancer Society; 2010

4. B. Chance, S. Nioka, J. Zhang, E. F. Conant, E. Hwang, S. Briest, S. G. Orel, M. D. Schnall, B. J. Czerniecki, "Breast cancer detection based on incremental biochemical and physiological properties of breast cancers: a six-year, two-site study," Acad. Radiol., 12(8), 925-933 (2005).

5. B. Jayachandran, J. Ge, S. Regalado, A. Godavarty, "Design and development of a hand-held optical probe towards fluorescence diagnostic imaging" J. Biomedical Optics 12(5), 054014-1 -10 (2007).

6. B.J. Tromberg, A. Cerussi, N. Shah, M. Compton, A. Durkin, D. Hsiang, J. Butler and Mehta., R., "Diffuse optics in breast cancer: detecting tumors in premenopausal women and monitoring neoadjuvant chemotherapy," Breast Cancer Res., 1(7), 279-285 (2005).

7. C. Xu, Q. Zhu, "Optimal probe design for dual-modality breast imaging." Proc. SPIE 6434, 64340B (2007).

8. D. Roetenberg,P. Slycke,A. Ventevogel,P.H. Veltink. "A portable magnetic position and orientation tracker." Sensors and Actuators A: Physical, vol. 135, Issue 2, (2007) $426-432$.

9. F. Bevilacqua, A.J. Berger, A.E. Cerussi, D. Jakubowski, B.J. Tromberg. "Broadband absorption spectroscopy in turbid media by combined frequencydomain and steady state methods." Appl Opt 2000; 39(34): 498-507.

10. F.H. Raab, et al., "Magnetic Position and Orientation Tracking System," IEEE Trans. Aerospace and Electronic Systems, vol. AES-15, no. 5, 1979, pp. 709-718.

11. J. Ge, B. Zhu, S. Regalado, A. Godavarty, "Three-dimensional fluorescenceenhanced optical tomography using a hand-held probe based imaging system", Med. Phys 35(7): 3354-63 (2008). 
12. J. Ge, S.J. Erickson, A. Godavarty, "Fluorescence tomographic imaging using a handheld-probe-based optical imager: extensive phantom studies," Applied Optics 48(33): 6408-6416 (2009).

13. J.N. Reed, "Impact of tracking system knowledge on multi-sensor 3D triangulation." SPIE 4714 April (2002).

14. J. Wang, B.W. Pogue, S. Jiang, K.D. Paulsen. "Near Infrared tomography of breast cancer hemoglobin, water, lipid, and scattering using combined frequency domain and CW measurement." Optics Letters 2010; 35(1).

15. K.S. No, and P. H. Chou, "Mini-FDPM and heterodyne mini-FDPM: handheld non-invasive breast cancer detectors based on frequency-domain photon migration," IEEE transactions on circuits and systems-I: regular papers, 52(12), 2672-2685 (2005).

16. L.V. Wang, H. Wu, Biomedical Optics Principles and Imaging. (2007)

17. N. Chen, M. Huang, H. Xia, D. Piao, E. Cronin, Q. Zhu, "Portable near-infrared diffusive light imager for breast cancer detection, ” J. Biomed. Opt., 9(3), 504510 (2004).

18. Q. Zhu, T. Durduran, V. Ntziachristos, M. Holboke, A.G. Yodh., "Imager that combines near-infrared diffusive light and ultrasound, " Opt. Lett., 24(15), 10501052 (1999).

19. S. Erickson, A. Godavarty, "Hand-Held Based Near-Infrared Optical Imaging Systems: A Review." Medical Engineering and Physics 2008 doi: 10.1016/j.medengphy.2008.10.004 (2008).

20. S.L. Meeks, W.A. Tome, T.R. Willoughby, P.A. Kupelian, T.H. Wagner, J.M. Buatti, F.J. Bova. "Optically Guided Patient Positioning Techniques." Seminars in Radiation Oncology 15 (2005): 192- 201.

21. S. Regalado, B. Zhu, J. Ge, S. J. Erickson, A. Godavarty, "Automated coregistered imaging using a hand-held probe-based optical imager," Rev. Sci. Instr. (in press) (2009).

22. V. Sao, K. Pourrezaei, A. Akin, H. Ayaz, "Breast tumor imaging using NIR LED based handheld continuous-wave imager." Proc. of the IEEE 29th Annual NE Bioeng. Conf., Reisman S., Foulds R., Mantilla B., eds. (2003), pp. 55-56. 


\section{APPENDIX}

\section{Appendix 1 Three Dimensional Tracking}

Triangulation Using Two Detectors for Tracking

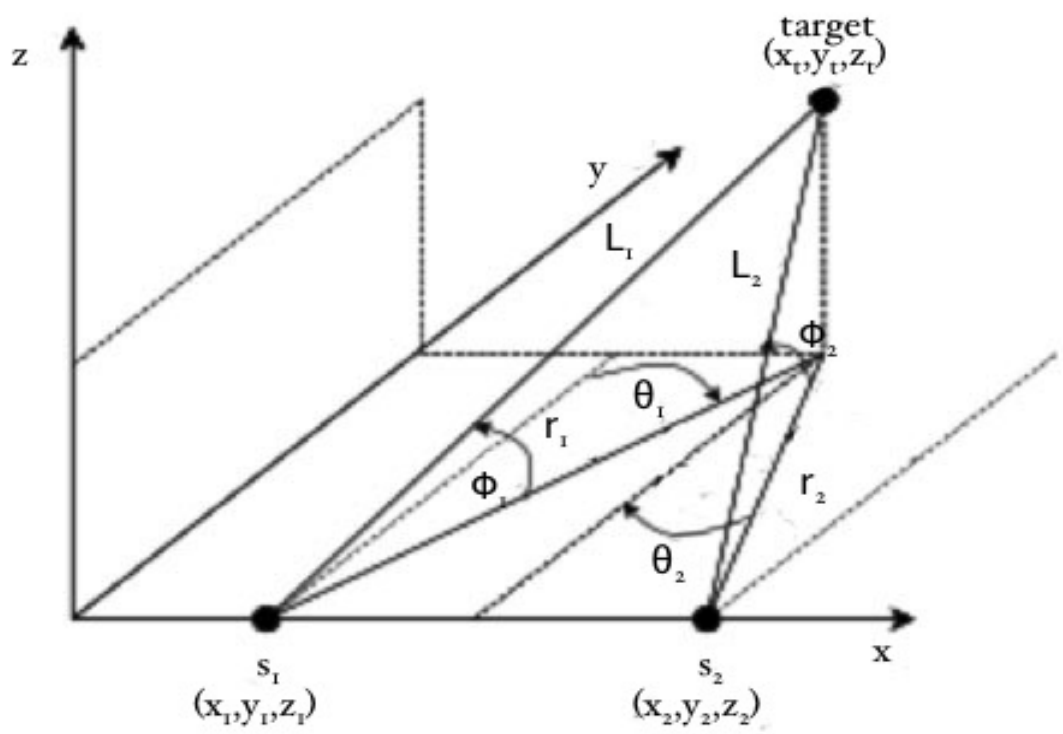

Figure 1 Coordinate System used to determine triangulation equations [Reed 2002]

The equations involved in the triangulation process are as given below: [Reed 2002]

$$
\begin{aligned}
& x_{t}=\left[x_{2} \tan \theta_{1}-x_{1} \tan \theta_{2}+\left(y_{1}-y_{2}\right) \tan \theta_{1} \tan \theta_{2}\right]\left(\tan \theta_{1}-\tan \theta_{2}\right)^{-1} \\
& y_{t}=\left[y_{1} \tan \theta_{1}-y_{2} \tan \theta_{2}+x_{2}-x_{1}\right]\left(\tan \theta_{1}-\tan \theta_{2}\right)^{-1} \\
& r_{i}=\sqrt{\left(x_{i}-x_{t}\right)^{2}+\left(y_{i}-y_{t}\right)^{2}} \\
& z_{t}=r_{i} \tan \phi_{i}+z_{i}
\end{aligned}
$$

In Figure $11 \mathrm{~s}_{1}$ and $\mathrm{s}_{2}$ are the detectors and the target is the LED being tracked. In the equations above, $\theta$ is the angle of rotation and $\phi$ is the angle of elevation of the point from the reference locations. $\mathrm{L}_{1}$ and $\mathrm{L}_{2}$ are the distance from the detectors to the target. Triangulation takes the angle at which the target is from the detectors at known locations and calculates the location of the target with respect to the detectors. The triangulation 
algorithm was written in MatLab and integrated to LabView using drivers for the Nintendo Wiimotes found on the National Instruments' website ${ }^{2}$. The drivers for the Nintendo Wiimote were created by a National Instruments programmer and made publicly available for no charge.

\section{Optical Tracker 3D Integration with the Automated Software}

Although the triangulation method was implemented to the 3D tracking software, it would not maintain its accuracy. Readily available software called Free-Track takes the Wiimote and uses it for head tracking in video games. The software gives accurate values for the 3D position of the LED's as well as pitch, yaw, and roll. LabView however, does not take inputs from software that is not LabView or MatLab based, so a method of integrating the software with LabView had to be found. A software package called Parallel Port Joystick was found which caused the output from the Wiimote in Freetrack to be read in Windows as a virtual controller. This can then be used in LabView as an input and the data can be used for tracking. A custom LabView GUI that takes the Wiimote as a joystick was created to allow for the data from the Free-Track software to be implemented into any other LabView or MatLab based software, such as the co-registration for $3 \mathrm{D}$ tracking using the Wiimote.

Similar to the $2 \mathrm{D}$ tracking, the files of the acoustic tracker were removed. The LabView subVI that was created to communicate directly with the Wiimote was integrated into the LabView automated software. The data was continuously collected

\footnotetext{
${ }^{2}$ http://forums.ni.com/ni/board/message?board.id=170\&thread.id=249428
} 
and converted to the appropriate numbers as in the 2D software. However, the outside software does the mathematics and LabView only displays these values for the position. As the numbers were collected, they were input to the co-registration files responsible for the co-registration of the image to the phantom mesh.

\section{Infrared LED configuration for 3D Tracking}

Tracking an object with the Wiimote requires the use of an infrared source to determine its location. In order to achieve different degrees of freedom (DOF), different configurations and numbers of sources can be used. For use with the Wiimote, a $950 \mathrm{~nm}$ LED would work, however, a single LED only gives 2D positioning of an object. Two LEDs have the possibility of 3D positioning and 2 degrees of rotation, thus only 5 DOF. In order to obtain $6 \mathrm{DOF}$ in $3 \mathrm{D}$ positioning 3 LEDs are necessary. If placed in a planar triangular shape the pitch and yaw will not be very accurate. For accurate 6 DOF tracking it is necessary for the peak of the triangular LED configuration to be placed at a depth that is larger than it is tall. In other words, the height of the triangle must be less than the depth at which the plane of the peak LED is from the base LEDs. To power the LEDs a single 1.5-volt AA battery will be used with the three LEDs connected in parallel. A switch will be used to turn the LEDs on and off when necessary and the LED assembly must be laced in contact with the hand held probe.

\section{Summary}

Although the 3D tracker was developed as discussed above and tested, the effort put forth in this area of the research was unsuccessful. By using the LED circuit created for the tracker and three or more LEDs, the 2D tracker, ideally, should be easily 
converted to a 3D tracker. However the mathematical theories for 3D tracking are limited, possibly by the hardware used for the optical tracker. There is also the possibility of using multiple ( 3 or more) Wiimotes to acquire multiple aspects of tracking. It is also necessary to define a reference point or some type of calibration points in which the operation of the LED will be contained within. This technique may need linear interpolation to determine a conversion formula from pixels to distance since equation 1 determined in Section 3.4.2 will not be useful in 3D tracking. Through linear interpolation the points defined by a calibration will allow the computer to determine a formula that is best for converting the pixels to distance depending on each study, since it is possible that the reference points change for each experiment. In addition, the $3 \mathrm{D}$ tracking will improve the automated software and co-registration approaches to have the ability to co-register collected images during in vivo studies. 